\title{
Coherent Radio Pulses From GEANT Generated Electromagnetic Showers In Ice
}

\author{
Soebur Razzaque, ${ }^{a}$ Surujhdeo Seunarine, ${ }^{b}$ \\ David Z. Besson, ${ }^{a}$ Douglas W. McKay, ${ }^{a}$ John P. Ralston, ${ }^{a}$ \\ and David Seckel ${ }^{c}$ \\ ${ }^{a}$ Department of Physics \& Astronomy \\ University of Kansas, Lawrence, KS 66045, USA \\ ${ }^{b}$ Department of Physics \\ University of Canterbury, Private Bag 4800, Christchurch, New Zealand \\ ${ }^{c}$ Bartol Research Institute \\ University of Delaware, Newark, DE 19716, USA
}

\begin{abstract}
Radio Cherenkov radiation is arguably the most efficient mechanism for detecting showers from ultra-high energy particles of $1 \mathrm{PeV}$ and above. Showers occuring in Antarctic ice should be detectable at distances up to $1 \mathrm{~km}$. We report on electromagnetic shower development in ice using a GEANT Monte Carlo simulation. We have studied energy deposition by shower particles and determined shower parameters for several different media, finding agreement with published results where available. We also report on radio pulse emission from the charged particles in the shower, focusing on coherent emission at the Cherenkov angle. Previous work has focused on frequencies in the $100 \mathrm{MHz}$ to $1 \mathrm{GHz}$ range. Surprisingly, we find that the coherence regime extends up to tens of Ghz. This may have substantial impact on future radio-based neutrino detection experiments as well as any test beam experiment which seeks to measure coherent Cherenkov radiation from an electromagnetic shower. Our study is particularly important for the RICE experiment at the South Pole.
\end{abstract}

\section{Contents}

\begin{tabular}{lll}
\hline 1 & Introduction & 2
\end{tabular}

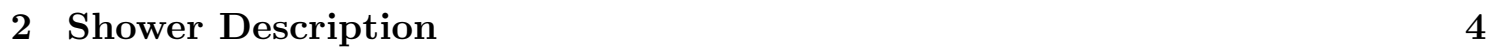

2.1 Energy Loss Mechanisms. . . . . . . . . . . . . . . . . . . . . . . . . . . . . 4

2.2 Longitudinal Profild. . . . . . . . . . . . . . . . . . . . . . . . . 5

2.3 Lateral Spread . . . . . . . . . . . . . . . . . . . . . . . . . 6

\begin{tabular}{lll}
\hline 3 & Shower Simulations & 7
\end{tabular}

3.1 Radiation Length $\ldots \ldots \ldots \ldots \ldots$. . . . . . . . . . . . 7 
$3.2 \quad$ Moliere radius . . . . . . . . . . . . . . . . . . . . . . . . . 8

3.3 Energy Loss in the Medium . . . . . . . . . . . . . . . . . . . . . . . . . . . 9

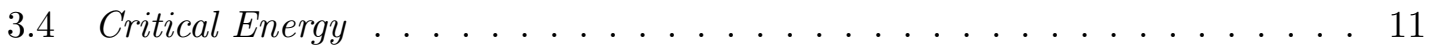

3.5 Track Lengths . . . . . . . . . . . . . . . . . . . . . . . . . . . . . . . . . 11

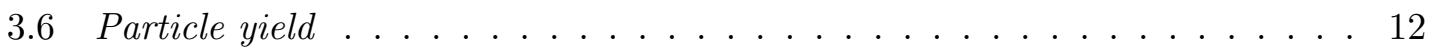

3.7 1-D Model Study . . . . . . . . . . . . . . . . . . . . . . . . . . . . 17

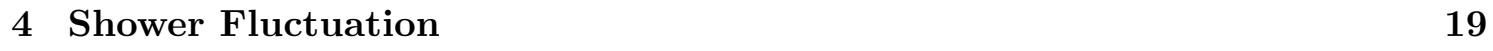

4.1 Fit to Simulations . . . . . . . . . . . . . . . . . . . . . . . . . . . . 19

\begin{tabular}{|lll}
5 & Electromagnetic Pulse Theory & 21
\end{tabular}

5.1 Fraunhoffer Limit. . . . . . . . . . . . . . . . . . . . . . . . . . . . . . . . . . . . . . 22

5.2 Parametrization Method . . . . . . . . . . . . . . . . . . . 25

$5.3 \quad$ Radiated Power and The Form Factor . . . . . . . . . . . . . . . . . . 26

$\begin{array}{lll}6 & \text { Electric Field Pulse Calculation } & 28\end{array}$

6.1 Vector Superposition . . . . . . . . . . . . . . . . . . . . . . 28

6.2 Phase Coherence . . . . . . . . . . . . . . . . . . . 28

6.3 Factorization of the Electric Field, and the Discrete Form Factor . . . . . . 31

6.4 The Discrete Form Factor and the Frequency Spectrum . . . . . . . . . . . . 32

6.5 The Analytic Form Factor and the Frequency Spectrum . . . . . . . . . . . . 32

6.6 Direct Calculation: Monte Carlo Field Spectrum . . . . . . . . . . . . . . . 34

6.7 Related Issues . . . . . . . . . . . . . . . . . . . . . . . . . 35

6.8 Angular Pulse Distribution . . . . . . . . . . . . . . . . . . . . . . . . . . 37

\begin{tabular}{lll}
\hline & Summary of Results and Conclusions & 39
\end{tabular}

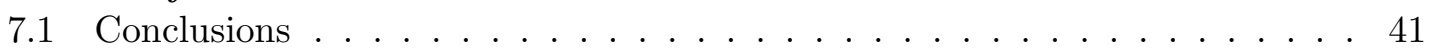

\section{Introduction}

Ultra high energy (UHE) neutrinos can travel without scattering over large distances. These may prove to be useful cosmological and astrophysical probes. They also present themselves as candidate high energy particles with which we can test the Standard Model of electro-weak theory beyond the energy regime of current accelerators. In an UHE electron-neutrino charged current interaction, the neutrino gives most $(\approx 80 \%)$ of its energy to the secondary electron, which can then initiate an electromagnetic cascade or shower. It was predicted that an electromagnetic shower generated by a high energy primary could develop a charge excess which would emit Cherenkov radiation coherently [1. For ultra high energy primaries the Cherenkov radiation would be coherent in the radio region of the spectrum; this long wavelength radiation might then be detected using radio antennas [2, 3, 迎. Given the small predicted flux of ultra high energy neutrinos [5, 6, 7], a suitable experiment to detect ultra high energy neutrinos using this method requires a large, dense (and radio-transparent) volume. Antarctic ice is suitable for this purpose. A detailed analysis of all aspects of such an experiment was done by Frichter, 
Ralston and McKay (FRM) [8], using a simulation developed by Zas, Halzen and Stanev (ZHS) [9]. FRM concluded that a modest array of optimally designed antennas could detect many events per year. The sensitivity of radio detection peaks above $1 \mathrm{PeV}$, which compliments an optical array such as AMANDA [10].

The Radio Ice Cherenkov Experiment (RICE) at the South pole [11] is designed as a prototype detector of ultra high energy neutrinos with energy $\geq \mathrm{PeV}$ using this method. One basic requirement for such an experiment is a reliable Monte Carlo simulation of the shower development, Cherenkov radiation, detector, and data acquisition system. One can also test the idea of coherent Cherenkov emission at accelerator facilities by dumping a beam of photons or electrons into a dense target like sand or salt or any other suitable medium. Such tests have begun with experiments at Argonne and SLAC [12]. A Monte Carlo simulation which can be easily adapted to such a test beam experiment, where Fresnel and possibly near zone radiation is important, and one that can include hadron showers conveniently, is clearly desirable. The ZHS simulation, designed for electromagnetic showers and Fraunhoffer (far zone) detection, has been a powerful tool. However an expansion and update with extensive testing, offering applications to test beam and neutrino astronomy, is currently needed.

We have written a GEANT-based Monte Carlo simulation to study coherent Cherenkov emission in ice, salt, or a beam dump. GEANT 3.21 [13] is a well known and widely used

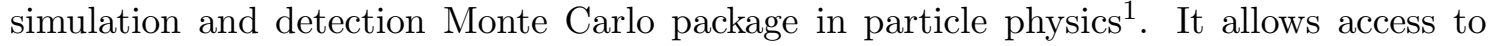
all details of the simulation such as controls of various processes, definition of target and detector media, and a complete history of all events simulated. GEANT can be used to simulate all dominant processes in $10 \mathrm{keV}-10 \mathrm{TeV}$ energy range, although it has not been extensively verified for energies $>100 \mathrm{GeV}$, where the extrapolation of well-known lowerenergy electromagnetic cross-sections becomes large, and other effects (LPM, e.g. [14, 15]) become significant. For electron energies above $10 \mathrm{GeV}$, GEANT uses screened BetheHeitler cross-section for bremsstrahlung together with the Migdal corrections [16, 17]. The first Migdal correction is important for energy $\geq 1 \mathrm{TeV}$, reducing the cross-section. The second correction reduces the differential cross-section for soft photon emission and is effective even at much lower energies, in the $100 \mathrm{MeV}-1 \mathrm{GeV}$ range. The LPM effect in the context of UHE electromagnetic shower development and radio emission has been discussed in [18, 19, 20].

GEANT is used to simulate electromagnetic showers inside materials from which we extract detailed track information including particle type, coordinates, energy and interaction time. From this track information, we investigate shower properties like radiation length, Moliere radius, critical energy and energy deposition in the material. We also determine the resulting radio pulse using standard electrodynamic calculations from charged particles' tracks and by parametrizing the shower. Ultimately we will consider hadronic shower information and GEANT provides the flexibility to expand our analysis to this case. We note that physics results presented thus far by RICE have neglected the hadronic shower contribution and are, in this respect, conservative.

\footnotetext{
${ }^{1}$ Differences between GEANT 3.21 and GEANT 4 are primarily at energies below the threshold for emission of Cherenkov radiation, and therefore do not affect the results presented here.
} 
The organization of this report is as follows. In section two, we discuss various aspects of electromagnetic showers and define quantities which characterize the shower. We present results on the shower structure from the Monte Carlo simulation in section three and compare them with established values in standard materials such as iron, lead and carbon. Our analysis includes the detailed breakdown of the radiation-generating charge imbalance into energy bins, the direct evaluation from energy considerations of Moliere radius and the determination from $\mathrm{d} E / \mathrm{d} x$ of radiation and energy deposition of the critical energy. We discuss shower-to-shower fluctuations by parametrizing the showers in section four. In section five, we review the theory behind coherent emission of an electromagnetic pulse from the shower [21]. In section six, we calculate the electric pulse from the shower using track information from GEANT. We summarize our results in section seven, making a number of comparisons with ZHS, and discuss future work.

\section{Shower Description}

When a high energy electron or photon hits a material target, an electromagnetic shower develops longitudinally inside the material. At the beginning of the shower, bremsstrahlung and pair production are the dominant processes. Due to these processes, the number of particles increases exponentially and the shower is created. Due to the dominance of bremsstrahlung as the primary energy loss mechanism for high energy electrons, the population of photons quickly dominates that of electrons or positrons. The energy of the initial particle is divided among the secondaries. The exponential production of particles is halted when the charged particles reach the critical energy $\left(E_{\mathrm{c}}\right)$. This is the transition region where ionization loss overtakes radiation loss as the most important electron energy loss mechanism. The particle population also reaches its maximum ("shower max") at this point. After reaching the critical energy, charged particles lose their energy predominantly inelastically by ionization, resulting in a subsequent decline in the number of particles in the shower as they degrade in energy and range out in the medium.

Multiple Coulomb scattering is responsible for the transverse spread of the shower. The shower core is populated by the highest energy particles. There is a long tail composed mostly of the coulomb- scattered low-energy component.

Other processes - Compton, Moller and Bhabha and positron annihilation build up a net charge (more electrons than positrons) in the shower as atomic electrons in the target medium are swept up into the forward moving shower.

\subsection{Energy Loss Mechanisms}

Energy loss of an electron due to radiation is well approximated by the Bethe-Heitler formula [22]

$$
-\left(\frac{\mathrm{d} E}{\mathrm{~d} x}\right)_{\mathrm{rad}}=E \frac{4 N_{\mathrm{o}} Z^{2} r_{e}^{2}}{137 A} \ln \left(\frac{183}{Z^{1 / 3}}\right)
$$

where $E$ is the initial energy of the electron, $x$ is the distance in g-cm ${ }^{-2}$ units, $N_{\mathrm{o}}$ is Avogadro's number, $A$ is the mass number of the nuclei of the medium, $Z$ is the atomic 
number of the medium and $r_{\mathrm{e}}^{2}=e^{4} / m^{2} c^{4}$ is the classical electron radius. For a composite medium like ice $\left(\mathrm{H}_{2} \mathrm{O}\right)$, one has to calculate the effective $Z$ and $A$ using the proportion by weight method?.

The Bethe-Bloch formula [23, 24] gives the energy loss due to ionization as

$$
-\left(\frac{\mathrm{d} E}{\mathrm{~d} x}\right)_{\text {ion }}=4 \pi N_{\mathrm{o}} \frac{Z}{A} r_{e}^{2} m c^{2}\left[\ln \left(\frac{2 m v^{2} \gamma^{2}}{I}\right)-1-\frac{\delta}{2}\right]
$$

where $I=10 Z \mathrm{eV}$ and $\gamma$ is the Lorentz factor in usual relativistic notation. At very high energy, one needs to take into account the density effect as the medium becomes polarized. This is taken care of by the following term inside the square bracket of Eq. (2):

$$
\delta / 2=\ln \left(\hbar \omega_{\mathrm{p}} / I\right)+\ln \beta \gamma-1 / 2,
$$

where $\omega_{\mathrm{p}}$ is the plasma frequency of the medium.

A rough expression for the critical energy, $E_{\mathrm{c}}$, can be found by the ratio of the expressions for radiation and ionization losses. The log term in Eq. (11) is roughly 4 and the square bracket term in equation (2) is approximately 11 if we include polarization effects. The ratio is then

$$
E_{\mathrm{c}} \approx \frac{605}{Z} \mathrm{MeV}
$$

An alternate definition of the critical energy is that of Rossi [25], according to which, the critical energy $\left(E_{\mathrm{c}}\right)$ is the energy at which the ionization loss per radiation length is equal to the electron energy. Critical energies determined from these two definitions are compared in a subsequent section.

The radiation length $\left(X_{\mathrm{o}}\right)$ is given by

$$
\frac{1}{X_{\mathrm{o}}}=\frac{4 N_{\mathrm{o}} Z^{2} r_{e}^{2}}{137 A} \ln \left(\frac{183}{Z^{1 / 3}}\right)
$$

so that the approximate expression for radiation loss (Eq. (11) becomes $-(\mathrm{d} E / \mathrm{d} x)=$ $E / X_{\mathrm{o}}$ or

$$
<E>=E_{\mathrm{o}} e^{-x / X_{\mathrm{o}}} .
$$

Eq. (6) serves as a definition of the radiation length; $X_{\mathrm{o}}$ is the thickness of material needed to reduce the mean energy of an electron to $1 / e$ of its original through bremsstrahlung.

\subsection{Longitudinal Profile}

Heitler [26] developed a simplified model of an electromagnetic shower according to which the initial electron, with energy $E_{\mathrm{o}}$, radiates a photon, of energy $E_{\mathrm{o}} / 2$, in the 1 st radiation length. In the 2 nd radiation length, the photon creates an electron-positron pair and the previous electron emits another photon; each particle now has energy $E_{\mathrm{o}} / 4$. This process continues until the critical energy $E_{c}$, defined in the previous section, is reached, at which point the shower is defined to be at maximum. At any radiation length, $t$, the

\footnotetext{
${ }^{2}$ see for example CONS110 section of [13].
} 
number of particles (electron, positron and photon) is $N(t)=2^{t}=\exp (t \ln 2)$ and the energy per particle is $E(t)=E_{\mathrm{o}} / 2^{t}$. Thus in this model the shower maximum occurs at $t_{\max }=\ln \left(E_{\mathrm{o}} / E_{c}\right) / \ln 2$ and the total number of particles at the maximum is $N_{\max }=$ $E_{\mathrm{o}} / E_{c}$. We can also calculate the integrated track length of the charged particles as $L=(2 / 3 \ln 2)\left(E_{\mathrm{o}} / E_{\mathrm{c}}\right)$.

Although this model is over-simplified in assuming equality of all the primary crosssections and in assuming that the shower is artificially cut off, it nevertheless provides a good description of the qualitative features of an electromagnetic cascade. Namely, it predicts that the total track length of the charged particles scales linearly, while the position of the shower maximum scales logarithmically with the initial energy of the shower. These are important consistency checks for the electromagnetic showers generated from any Monte Carlo.

An analytic method for realistic shower development was created by Carlson and Oppenheimer [27] and later extended by Landau and Rumer [28]. Analytic shower equations are solved in two approximations, namely approximation $A$ and approximation $B$ [25]. One neglects the Compton effect and ionization loss in approximation A. One includes a constant energy loss per radiation length for all electrons and positrons in approximation $B$, which is therefore expected to be a better model of the data. The derivation of the integral spectra or number of particles (electrons and positrons) at different shower depth $t$ in approximation $\mathrm{A}$ and $\mathrm{B}$ can be found in references [25, 29].

Greisen first parametrized the longitudinal profile of a photon-induced electromagnetic air shower [30]. The Greisen parametrization (GP) is based on analytic shower theory in approximation $\mathrm{A}$. The difference between the more realistic approximation $\mathrm{B}$ and approximation $\mathrm{A}$ is that of a slowly increasing function of the age parameter $(s)$. The number of particles in a given energy range increases when $s<1$, reaches a maximum when $s=1$ and then declines when $s>1$.

Hillas [31] later modified Greisen's parametrization to fit Monte Carlo simulation of 0.1-1 TeV photon-induced showers. This modified GP was later used by Fenyves et. al. [32] and is given for a single electron or photon of energy $E_{\mathrm{o}}$ as:

$$
N^{(\pi)}\left(E_{\mathrm{o}}, E, t\right)=\frac{0.31 A(E)}{\sqrt{y}} \exp \left[t_{1}\left(1-1.5 \ln s_{1}\right)\right]
$$

where $t_{1}$ is the modified depth defined to be $t_{1}=t+a_{\pi, \gamma}(E)$ and $s_{1}$ is the modified age parameter defined to be $s_{1}=3 t_{1} /\left(t_{1}+2 y\right)$. Superscript $\pi$ denotes the total electrons and positrons following the convention in the literature. The parameter $y$ called lethargy is defined to be $y=\ln \left(E_{\mathrm{o}} / E_{\mathrm{c}}\right)$, where $E_{\mathrm{c}}$ is the critical energy. One finds the parameters $A(E), a_{\pi}(E)$ for electron-induced and $a_{\gamma}(E)$ for photon-induced showers by fitting Monte Carlo simulations.

\section{$2.3 \quad$ Lateral Spread}

The transverse development of the shower is well described by a quantity called Moliere radius $\left(R_{\mathrm{M}}\right)$. It is determined by the average angular deflection per radiation length at the

\footnotetext{
${ }^{3}$ Shower $\operatorname{depth} t=\operatorname{depth} / X_{\mathrm{o}}$ is always given in terms of the radiation length $\left(X_{\mathrm{o}}\right)$.
} 
critical energy $\left(E_{\mathrm{c}}\right)$ due to multiple Coulomb scattering. The average deflection is given by [33]:

$$
<\delta \theta^{2}>=\left(\frac{E_{\mathrm{s}}}{E}\right)^{2} \delta t
$$

where $E_{\mathrm{s}}=\sqrt{4 \pi / \alpha} m_{\mathrm{e}} c^{2}=21.21 \mathrm{MeV}$ is the scale energy.

A numerical estimate of $R_{\mathrm{M}}$ is obtained by considering the fraction of energy that escapes transverse to the shower axis [34, 35]:

$$
\frac{U(r)}{E}=\frac{\int_{0}^{\infty} \int_{r}^{\infty} E(r, t) \mathrm{d} r \mathrm{~d} t}{\int_{0}^{\infty} \int_{0}^{\infty} E(r, t) \mathrm{d} r \mathrm{~d} t}
$$

where energy $(E)$ is expressed as a function of shower depth $(t)$ and radial distance $r$ from the shower axis. By definition, ninety percent of the shower energy is contained inside a cylinder of radius $R_{\mathrm{M}}$ centered on the shower axis. I.e., $r=R_{\mathrm{M}}$ when $U(r) / E=0.1$ in Eq. (9). Moliere radius is independent of the energy of the shower and depends only on the tracking medium in general.

In Rossi's definitions, the Moliere radius is related to the critical energy $\left(E_{\mathrm{c}}\right)$ and radiation length $\left(X_{\mathrm{o}}\right)$ of the material [34, 35] through the equation

$$
R_{\mathrm{M}}=\frac{X_{\mathrm{o}} E_{\mathrm{s}}}{E_{\mathrm{c}}}
$$

which follows from Eq. (8).

\section{Shower Simulations}

The target medium in our GEANT simulations, unless otherwise stated, is defined to be an ice cube of side 1 kilometer. Given the molecular composition, GEANT calculates the effective atomic number, $Z=7.2$ and an effective mass $A=14.3$ for the compound ice. Other parameters like radiation length, absorption length and cross-sections are calculated automatically, once $A$ and $Z$ are specified.

GEANT gives all the details of particle tracking information like interaction points, total energy, energy lost in interaction, interaction time and so on. We used double precision for all the variables in our output data files to minimize roundoff errors. Unless stated otherwise, all particles are tracked down to total energy of $0.611 \mathrm{MeV}$, which is lower than the energy at which particles are still relativistic and emit Cherenkov radiation.

\subsection{Radiation Length}

The radiation length given by Eq. (5) depends on the atomic and mass numbers of the material. However employing the definition given by Eq. (6), we can fit an exponential to the average energy of the injected electron at increasing depths. The average is taken from the data of a number of showers generated by the Monte Carlo. Our ability to recover the input value of $X_{\mathrm{o}}$ will serve as an internal consistency check to ensure that we are tracking all the particles of interest, along with their energies. 


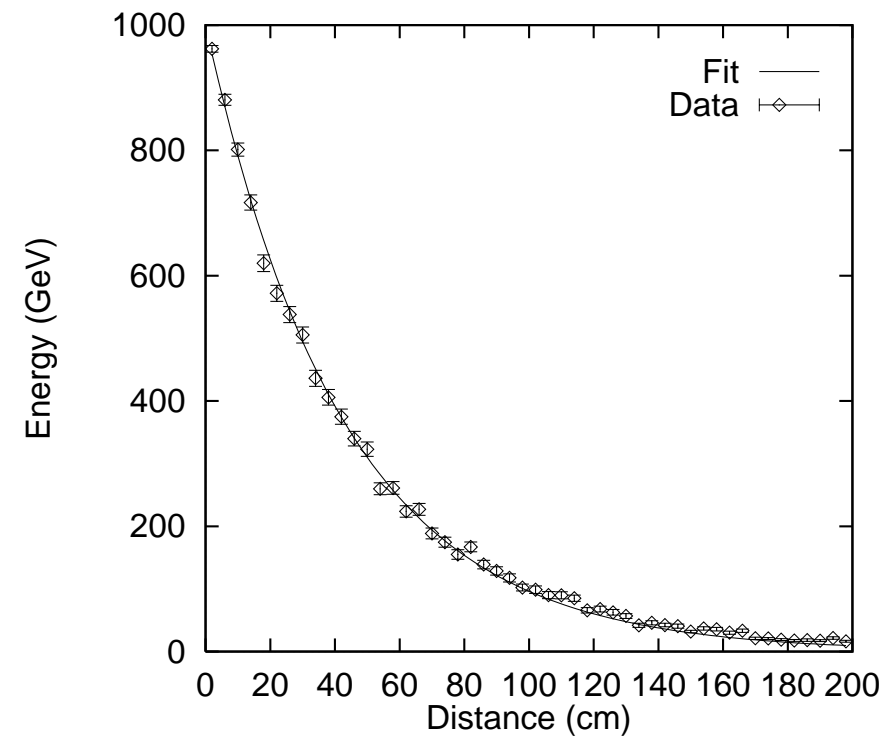

Figure 1: Average energy of a $1 \mathrm{TeV}$ electron injected in ice vs. distance in cm. Monte Carlo data points are obtained from 500 electron tracks and the errorbars correspond to standard error or $s / \sqrt{ } N$, where $s$ is the standard deviation and $N$ is the number of tracks (500). The solid line is the exponential fit as in Eq. (6) with $E_{\mathrm{o}}=1 \mathrm{TeV}$. This fit gives a radiation length of $42.2 \pm 4.3$ cm. A $100 \mathrm{MeV}$ energy threshold was used in all 500 simulations.

We generated 500 electron tracks each with $1 \mathrm{TeV}$ primary energy $\left(E_{r m o}\right)$ incident on the ice target and calculated energy loss due to bremsstrahlung. We tracked all the electrons down to energy $100 \mathrm{MeV}$, well above the value of the critical energy so that bremsstrahlung is dominant. We show the energy of the injected electron, averaged over the 500 tracks, as a function of distance in Fig. 1. The errorbars correspond to standard error or $s / \sqrt{ } N$, where $s$ is the standard deviation and $N$ is the number of tracks (500). A least squares fit of Eq. (6) to the Monte Carlo data keeping fixed $E_{\mathrm{o}}=1 \mathrm{TeV}$ yields a radiation length of $42.2 \pm 4.3 \mathrm{~cm}$. The confidence level (CL) for the fit is $95.7 \%$.

Given the molecular composition, GEANT also calculates the medium's radiation length from the standard formula. For ice, GEANT calculates $X_{\mathrm{o}}=38.8 \mathrm{~cm}$, which is roughly in agreement with the value we extract by tracking bremsstrahlung photons.

\subsection{Moliere radius}

To calculate the Moliere radius, we construct an imaginary cylinder centered on the shower axis (up to the physical length of the shower). We add the energies, $U$, of all the tracks that leave the cylinder without re-entering. By varying the radius of the cylinder we obtain $R_{\mathrm{M}}$, which is the radius of the cylinder when the fraction $U / E_{\mathrm{o}}$ is equal to 0.1 .

The Moliere radii $\left(R_{\mathrm{M}}\right)$ for lead and iron are found to be $1.6 \mathrm{~cm}$ and $2.1 \mathrm{~cm}$, respectively (see Fig. 2). The value for lead is in agreement with experimental value of $1.6 \mathrm{~cm}$ [34]. We did not find the corresponding value for iron in the literature. A similar analysis for ice results in $R_{\mathrm{M}}^{\mathrm{ice}}=13 \pm 1 \mathrm{~cm}$. We quote the standard error, defined as $s / \sqrt{ } N$, where $s$ is 

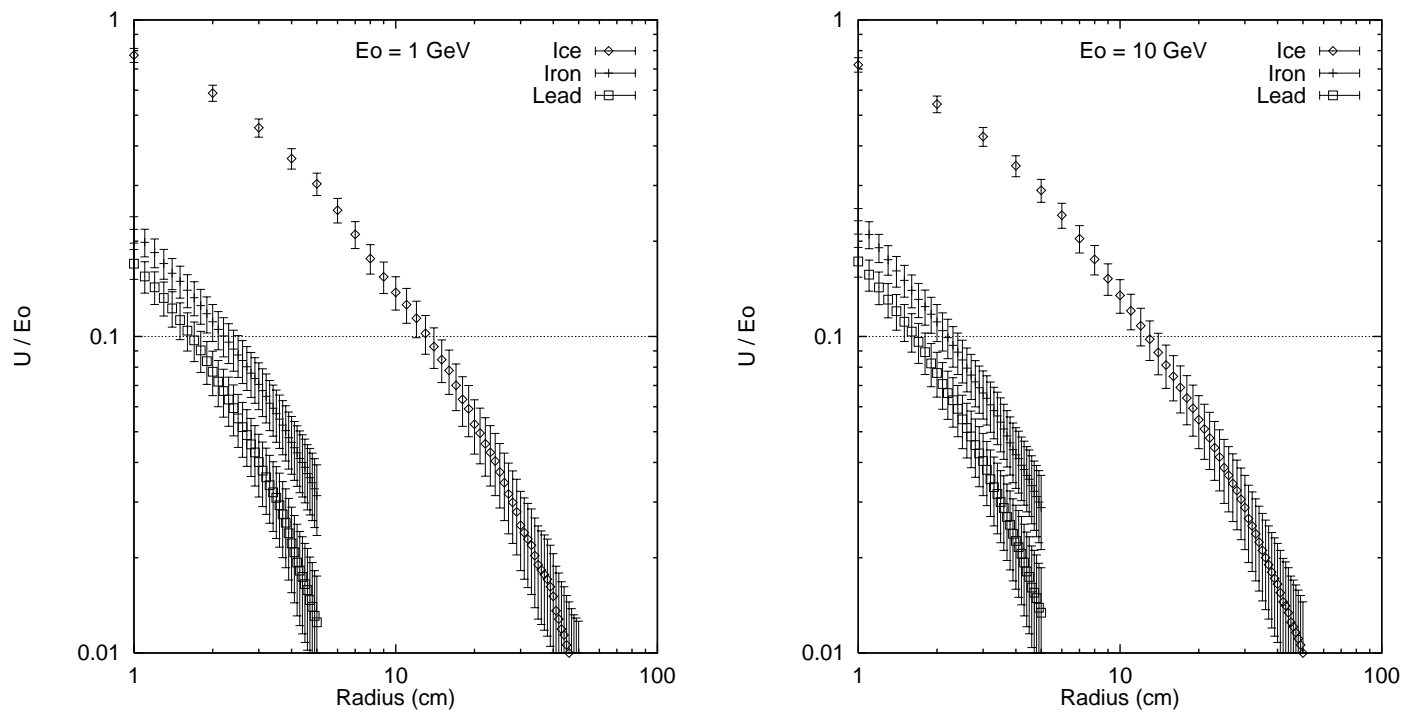

Figure 2: Moliere radius corresponds to the transverse development of the shower. When the fraction $U / E_{\mathrm{o}}$ (the ratio of total energy inside an imaginary tube along the showers axis to the initial energy of the shower) is 0.1 (the horizontal straight line), the corresponding radius is by definition, the Moliere radius for that material. It depends on the material and not on the energy of the shower. Here we compare showers of energies $1 \mathrm{GeV}$ (left plot) and $10 \mathrm{GeV}$ (right plot) for each material; the Moliere radius is observed to be independent of energy in this range.

the standard deviation and $N$ is the number of showers. It should be noted that the error bars are correlated from bin to bin.

\subsection{Energy Loss in the Medium}

The signal from a Cherenkov type detector is proportional to the track length, which is itself proportional to the energy deposition of the shower particles in the medium. Therefore, we study the ionization loss per unit length in this section.

To determine $\mathrm{d} E / \mathrm{d} x$ due to ionization from Monte Carlo, we generated 500 separate $5 \mathrm{GeV}$ electron tracks in ice and kept a record of the rate at which energy was lost due to ionization. The dots in Fig. 3 (left plot) shows $\mathrm{d} E / \mathrm{d} x$ in ice for the 500 tracks at different Monte Carlo steps. We also calculated $\mathrm{d} E / \mathrm{d} x$ ionization loss in carbon, which has roughly the same atomic weight as ice, as a consistency check. The result is shown in Fig. 3 (right plot).

The average $\mathrm{d} E / \mathrm{d} x$ curve from GEANT simulation matches the analytic approximation, Eq. (2) as shown in Fig. 19. The average ionization loss in ice (left plot) in the relativistic region is approximately $2.4 \mathrm{MeVg}^{-1} \mathrm{~cm}^{-2}$ and the average minimum ionization loss is approximately $1.9 \mathrm{MeVg}^{-1} \mathrm{~cm}^{-2}$. We have also calculated the average $\mathrm{d} E / \mathrm{d} x$ ionization loss in carbon (right plot) as a consistency check. 

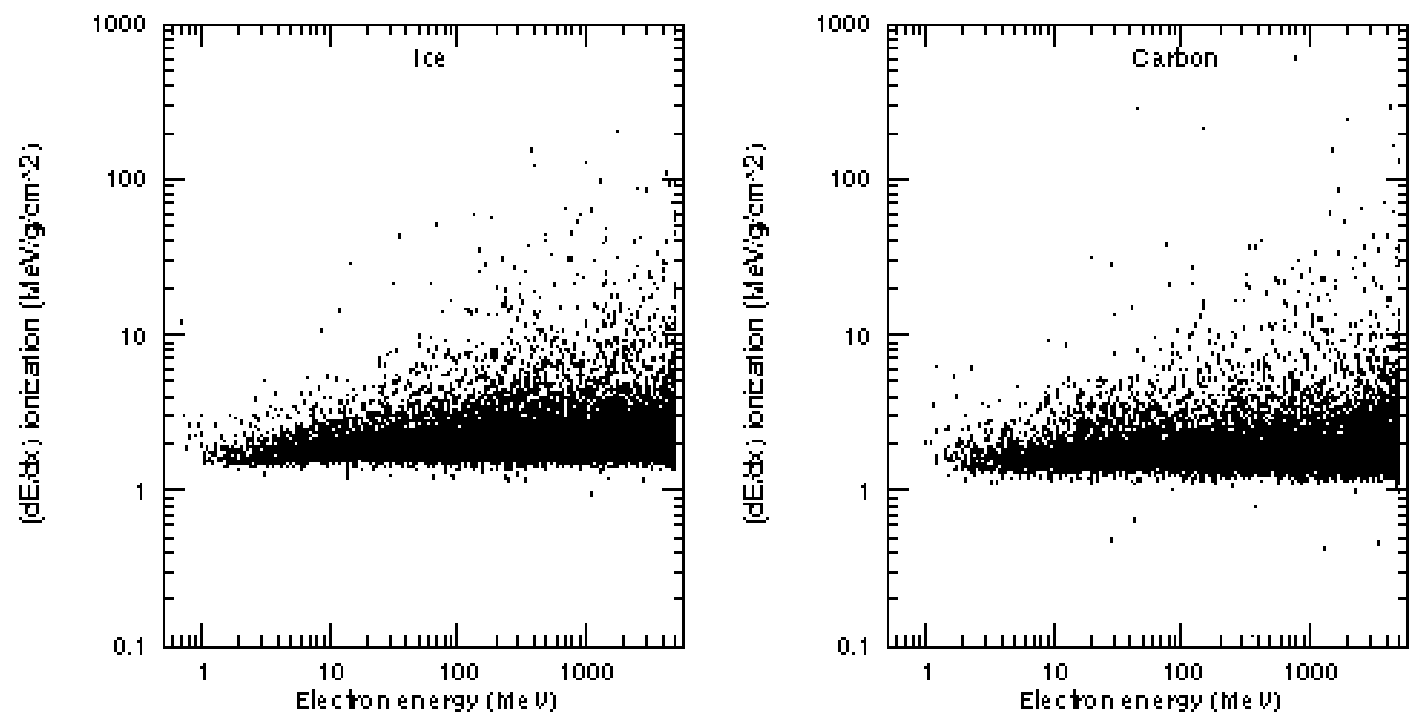

Figure 3: Electron stopping power or $\mathrm{d} E / \mathrm{d} x$ ionization loss in ice (left plot) and in carbon (right plot). The dots correspond to energy lost per unit length at each Monte Carlo step of 500 electron tracks each with $5 \mathrm{GeV}$ primary energy.
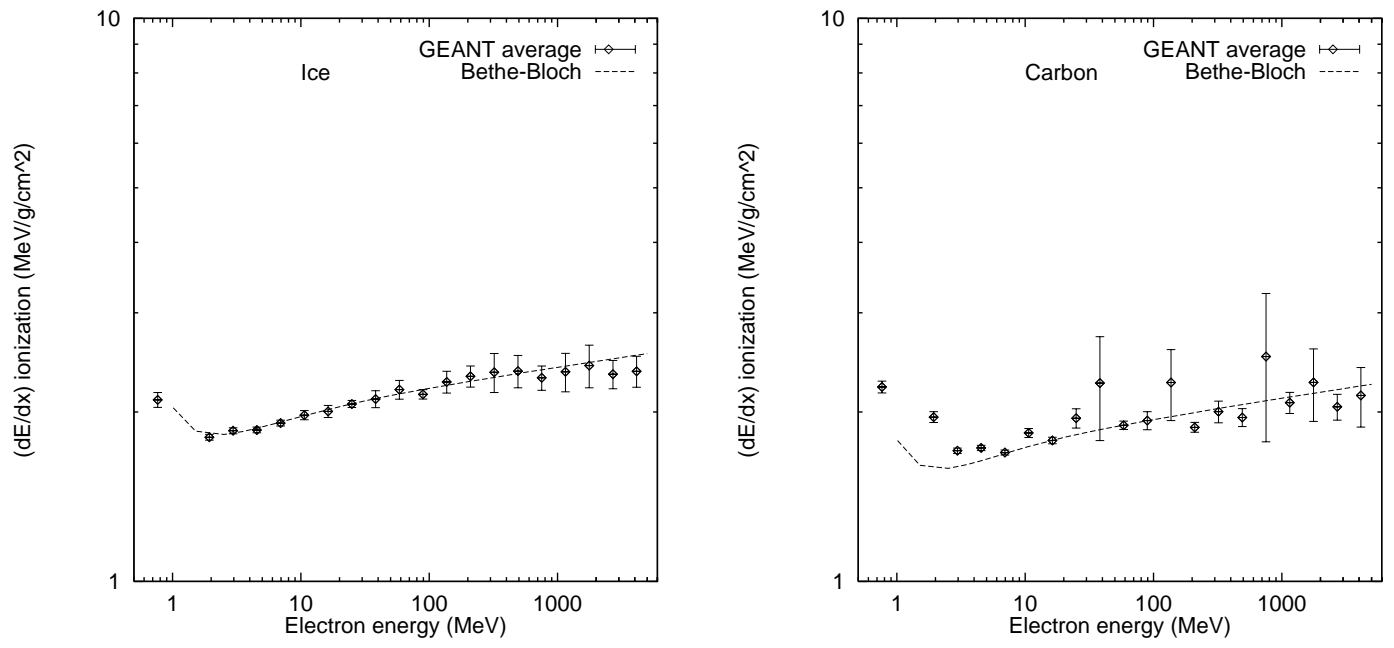

Figure 4: Average electron stopping power or $\mathrm{d} E / \mathrm{d} x$ ionization loss in ice (left plot) and in carbon (right plot) calculated from GEANT data in Fig. 3. The error bars correspond to standard error or $s / \sqrt{ } N$, where $s$ is the standard deviation and $N$ is the number of tracks (500 in this case). The solid lines are analytic curves calculated from Eq. (2). The agreement between Monte Carlo and the analytic formula is reasonably good in the relativistic rise region. 


\subsection{Critical Energy}

There are several methods to calculate the critical energy $\left(E_{\mathrm{c}}\right)$. The simplest is of course, the rough estimate in Eq. (何). One can also obtain a value for $E_{\mathrm{c}}$ in the Rossi definition from Eq. (10) after supplying the value of the Moliere radius $\left(R_{\mathrm{M}}\right)$ and the radiation length $\left(X_{\mathrm{o}}\right)$ found earlier. Yet another method is to find the energy at which the $\mathrm{d} E / \mathrm{d} x$ curves due to ionization and radiation losses intersect, which is the Monte Carlo equivalent of the analytic expression in Eq. (牙).

First, we find the critical energy from the Rossi definition using Eq. (10). The Moliere radius for ice was found to be $R_{\mathrm{M}}^{\text {ice }}=13 \pm 1 \mathrm{~cm}$ and the radiation length, $X_{\mathrm{o}}=42.2 \pm 4.3$ $\mathrm{cm}$. The critical energy is then calculated from Eq. (10) to be $E_{\mathrm{c}}^{\text {ice }}=68.8 \pm 8.8 \mathrm{MeV}$. Rossi [25] quotes $65 \mathrm{MeV}$ for the critical energy of water, in agreement with our calculated value. As a consistency check, we also calculated the critical energy for lead using the Moliere radii found from GEANT simulation (section 3.2). The result is $E_{\mathrm{c}}^{\text {lead }}=7.4 \mathrm{MeV}$, where we have used the radiation length of lead to be $0.56 \mathrm{~cm}$. This is in agreement with the experimental result [36].

Second, we calculate the energy loss due to radiation according to the formula in Eq. (6) using the radiation length $X_{\mathrm{o}}=42.2 \pm 4.3 \mathrm{~cm}$ we found before. We then find the energy at which this line crosses the ionization loss curve (see Fig. 5). The value for critical energy (the energy at the crossing point of these two curves) found in this method is $90 \pm 9 \mathrm{MeV}$, where the error includes both the standard error in the $\mathrm{d} E / \mathrm{d} x$ points and the uncertainty in the radiation loss due to the error in $X_{\mathrm{o}}$. The approximate formula (Eq. (田) gives $E_{\mathrm{c}}=84 \mathrm{MeV}$, close to what we found from simulation. The critical energy calculated in this way gives a higher value than Rossi definition, as expected.

\subsection{Track Lengths}

If we consider all processes to be elastic except ionization, then an estimate of the upper bound for the total track length is given by,

$$
L=E_{\mathrm{o}} /\left(\frac{\mathrm{d} E}{\mathrm{~d} x}\right)_{\text {ion }}^{\min }
$$

where $(\mathrm{d} E / \mathrm{d} x)_{\text {ion }}^{\min }$ is the minimum ionization energy loss per unit length. This value is about $1.9 \mathrm{MeVg}^{-1} \mathrm{~cm}^{-2}$ for ice, (see Fig. $\$$ ) which yields a maximum total track length of $\sim 1350$ radiation lengths or 570 meters in ice for a $100 \mathrm{GeV}$ shower. The actual total track length is less than this value, since the energy loss due to ionization increases in the relativistic region.

We plot different track lengths in Fig. 6 for a $100 \mathrm{GeV}$ shower (averaged over 25 showers) versus the kinetic energy threshold used in the Monte Carlo to generate them. The total track length for electrons and positrons together is denoted by total absolute track length, the sum of electron and positron track-lengths projected along the shower axis is denoted by total projected $(e+p)$ track length and the difference between the electron and positron track lengths projected along the shower axis is denoted by total projected $(e-p)$ track length. As we increase the threshold, so the simulation neglects 


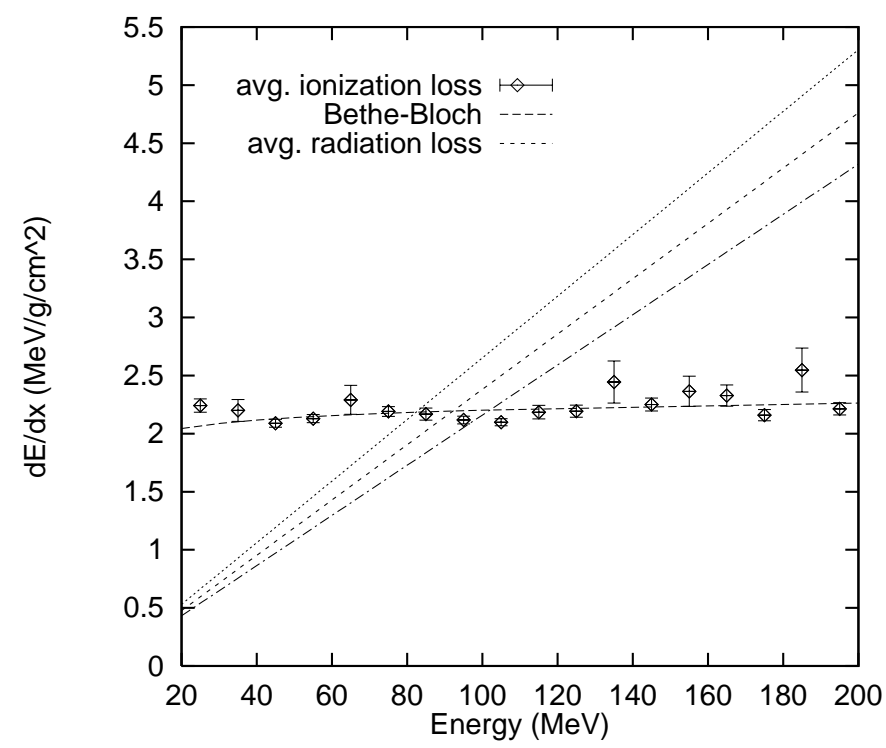

Figure 5: Radiation and ionization energy loss vs. electron energy plot from GEANT Monte Carlo simulation. The diagonal straight lines correspond to energy loss due to radiation with radiation length $X_{\mathrm{o}}=42.2 \pm 4.3 \mathrm{~cm}$, where the lowest curve corresponds to $X_{\mathrm{o}}=42.2+4.3 \mathrm{~cm}$ and the highest to $X_{\mathrm{o}}=42.2-4.3 \mathrm{~cm}$. The average ionization energy loss data are plotted as obtained before (see Fig. (1). The error bars show the standard errors on the $\mathrm{d} E / \mathrm{d} x$ points. The crossing point of the two curves gives one definition of the critical energy.

track-lengths from low energy particles, we expect these total calculated track-lengths to decrease.

We have also compared our results to those obtained using the ZHS Monte Carlo in Fig. 6. Although the qualitative behavior of this scaling is the same for both Monte Carlos, ZHS track lengths are consistently higher than GEANT track lengths by about $50 \%$. In particular, GEANT produces an absolute track length of 400 meter for $0.1 \mathrm{MeV}$ kinetic energy threshold or $0.611 \mathrm{MeV}$ total energy threshold. ZHS on the other hand produces 650 meters of absolute track length for the same threshold, which is significantly above our estimated maximum of 570 meters, based on the GEANT generated $100 \mathrm{GeV}$ data shown in Fig. 6 and application of Eq. (11).

The total track lengths increase with shower energy as more particles are created. The track lengths are expected to increase linearly with energy for a given a energy threshold used in the Monte Carlo. This scaling is shown in Fig. 0 below. Straight line fits to the absolute track length and the projected $(e-p)$ track length are also plotted. The slopes of those straight lines are 3.2 and 0.5 respectively.

\subsection{Particle yield}

To generate a longitudinal profile of the shower, one counts the number of particles crossing planes perpendicular to the shower axis inside the ice. We have calculated profiles for both the total number of particles $(e+p)$ and for the excess charge $(e-p)$ in the shower. 


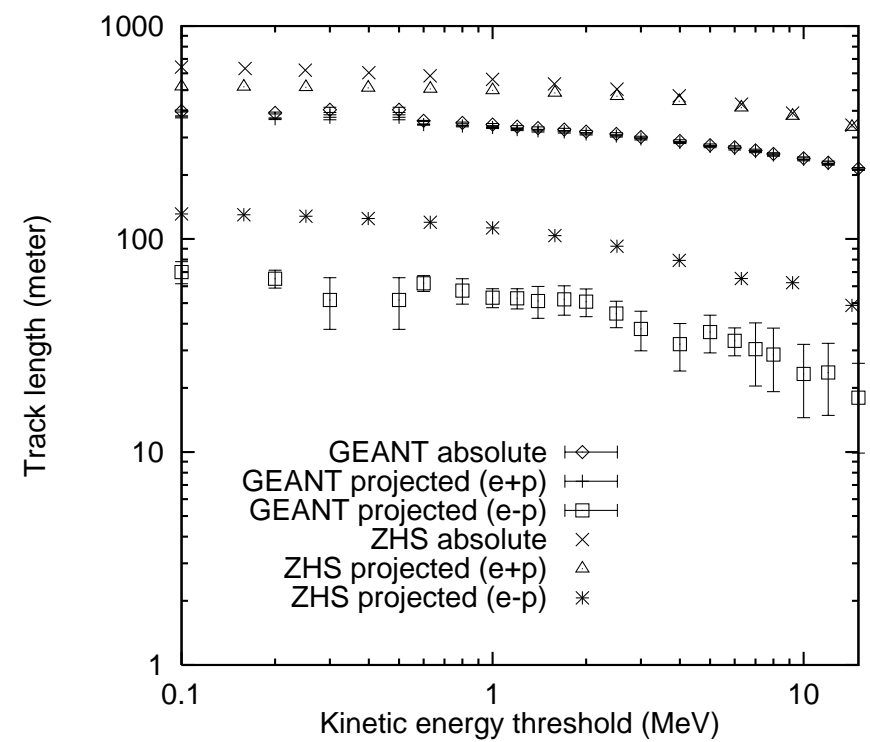

Figure 6: Total track lengths as functions of the kinetic energy threshold used in the Monte Carlos. Here we show the total absolute track-lengths, the total $(e+p)$ track-lengths projected along the shower axis and the total $(e-p)$ track-lengths projected along the shower axis. The analysis is done for a $100 \mathrm{GeV}$ shower (averaged over 25 showers) using the GEANT and ZHS Monte Carlo simulations.

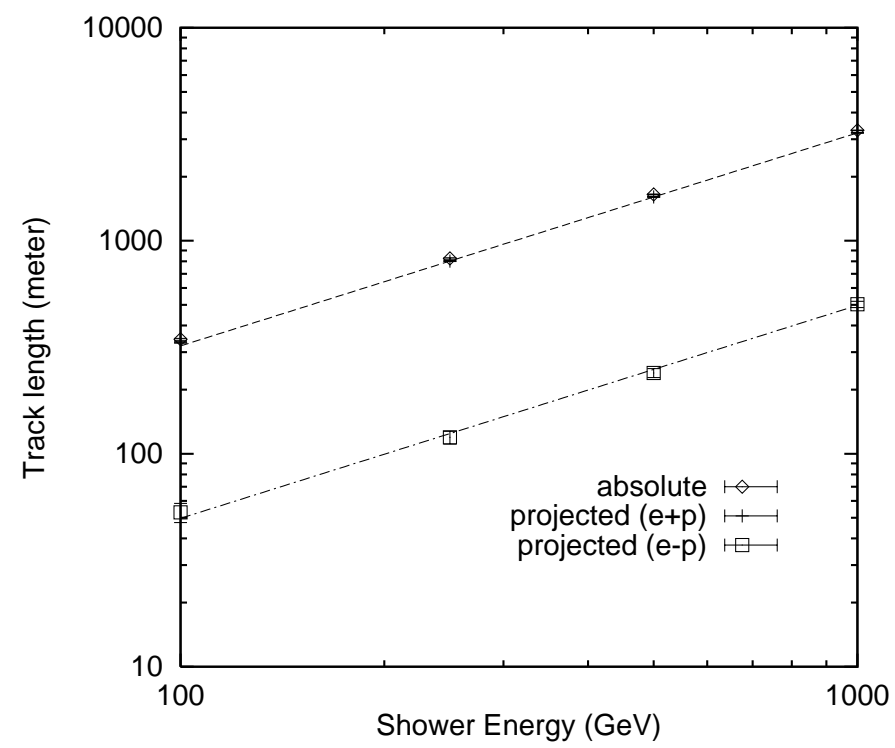

Figure 7: Total absolute track length, total projected $(e+p)$ track length and total projected $(e-p)$ track length as functions of shower energy from GEANT simulations. The linear scaling of the track lengths with the shower energy is clearly observed. We used a kinetic energy threshold of $1 \mathrm{MeV}$ (see Fig. 6) and averaged over 25 showers in each case. 


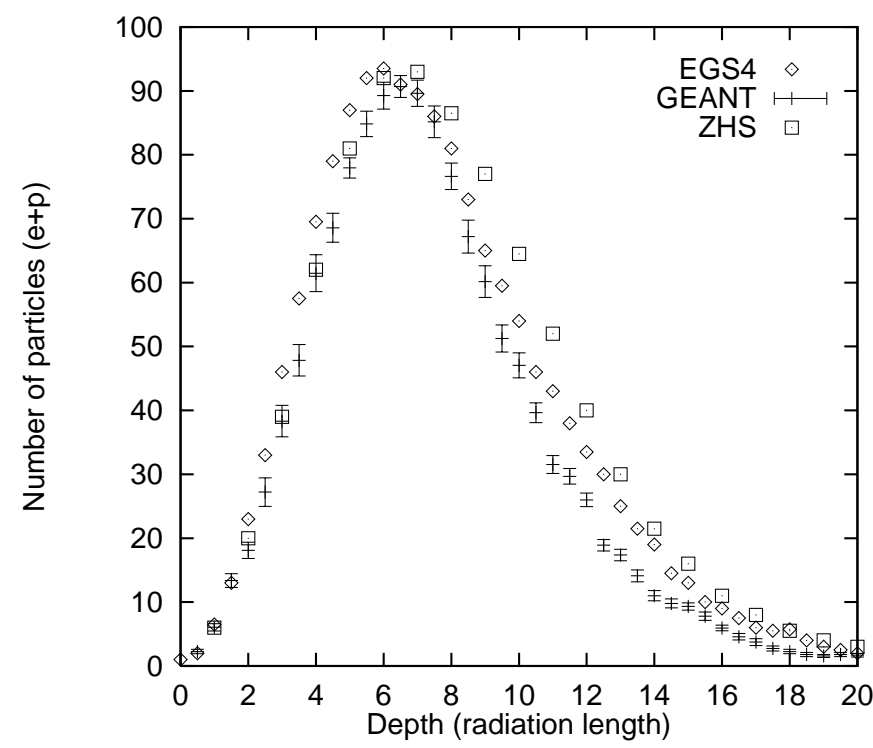

Figure 8: The longitudinal profiles of a $30 \mathrm{GeV}$ shower (averaged over 50 showers) in iron simulated by GEANT. The longitudinal profile is calculated by counting the number of electrons and positrons crossing planes on the shower axis every half radiation length with total energy greater than $1.5 \mathrm{MeV}$. We also plot the longitudinal profiles of the same shower from EGS4 (from PDG 2000 [37]) and by ZHS Monte Carlos.

As a consistency check of GEANT, we have simulated $30 \mathrm{GeV}$ electron-induced showers in iron and compared the profiles with the modified Greisen parametrization, Eq. (7). The longitudinal profiles were obtained by adding the number of particles with total energy greater than $1.5 \mathrm{MeV}$, crossing planes spaced one-half radiation length apart, and perpendicular to the shower axis (see Fig. 8). The number of particles agrees reasonably well with EGS4, an electromagnetic shower code developed at SLAC which simulates the same shower [37]. The total number of particles from ZHS simulation is also shown in the plot.

We have fitted the GEANT generated electromagnetic showers in ice by the modified GP in Eq. (77). We used the value for critical energy $\left(E_{\mathrm{c}}\right)$ in ice to be $67.7 \mathrm{MeV}$. The fitting parameters and the confidence level (CL) of the fits for showers of energy $100 \mathrm{GeV}$ (averaged over 100 showers), $500 \mathrm{GeV}$ (averaged over 50 showers) and $1 \mathrm{TeV}$ (averaged over 20 showers) each with $5 \mathrm{MeV}$ threshold energy are given in Table 1 for both electron and photon primaries. The fits to longitudinal profiles are plotted in Fig. 9 . 

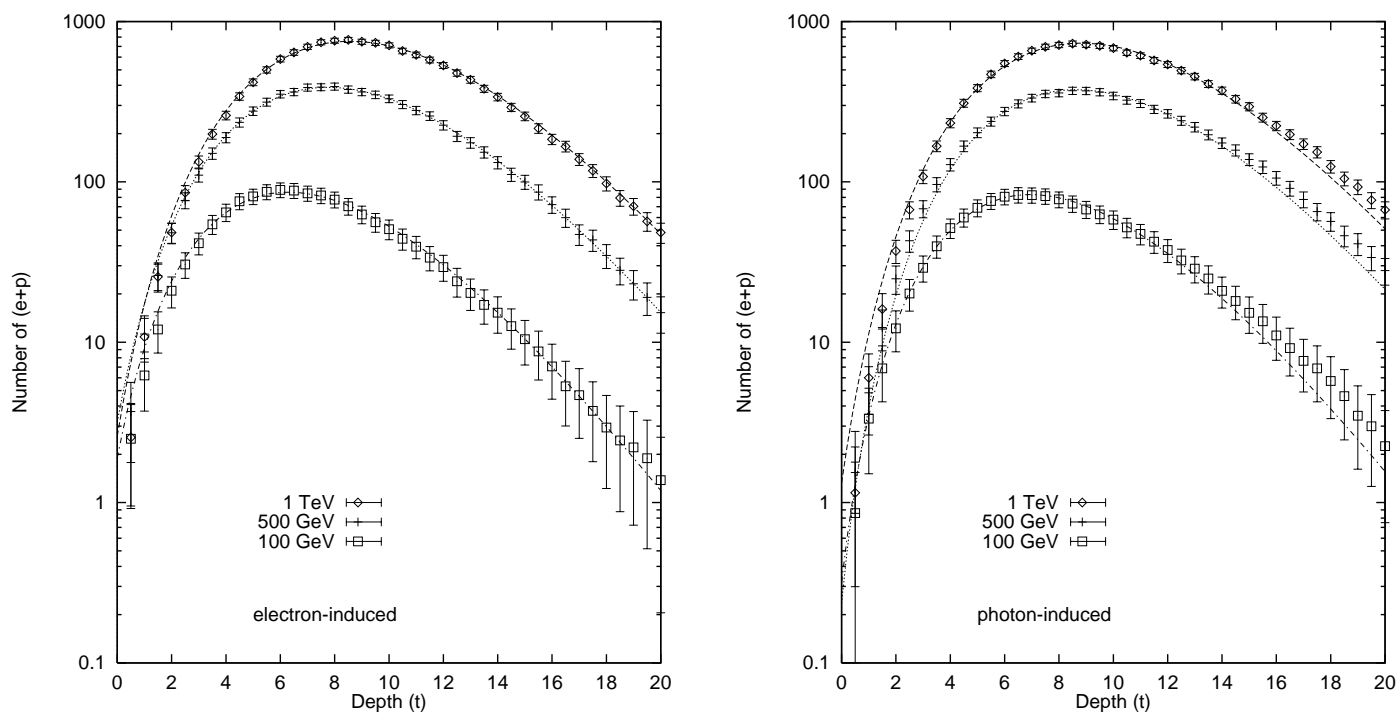

Figure 9: Longitudinal profiles of electron-induced (left) and photon-induced (right) showers in ice of primary energy $1 \mathrm{TeV}, 500 \mathrm{GeV}$ and $100 \mathrm{GeV}$, averaged over 20, 50 and 100 showers respectively. The threshold energy used is $5 \mathrm{MeV}$. The error-bars correspond to standard error. The smooth curves are Greisen parametrization fits as given in Eq. (7). The fitting parameters and the confidence levels (CL) for the fits are given in Table 1. The critical energy for ice used in the fits is $68.8 \mathrm{MeV}$.

Table 1: Greisen parameters for GEANT showers in ice with $5 \mathrm{MeV}$ threshold and

\begin{tabular}{c|cccc}
\multicolumn{5}{c}{$E_{\mathrm{c}}=68.8 \mathrm{MeV}$. } \\
\hline \hline Primary & $E_{o}(\mathrm{GeV})$ & $A(E)$ & $a_{\pi, \gamma}$ & $\mathrm{CL}(\%)$ \\
\hline \multirow{3}{*}{$\gamma$} & 100 & 0.50 & 0.33 & 96.3 \\
& 500 & 0.50 & 0.26 & 81.9 \\
& 1000 & 0.50 & 0.76 & 71.8 \\
\hline \multirow{3}{*}{$e^{-}$} & 100 & 0.52 & 0.99 & 95.6 \\
& 500 & 0.51 & 1.14 & 95.8 \\
& 1000 & 0.52 & 1.01 & 88.4 \\
\hline \hline
\end{tabular}

The parameter $A(E)$ gives over-all normalization for the number of particles. We see from Table 1 that the number of particles at the shower maximum for a photon-induced shower $(A(E)=0.50)$ is slightly less than that of an electron-induced shower $(A(E)=$ 0.52). The other parameter $a_{\pi, \gamma}$ takes care of the shift of the whole shower between a photon-induced $\left(a_{\gamma}\right)$ and an electron-induced $\left(a_{\pi}\right)$ shower. This shift is approximately 0.7 radiation length.

We have also fitted GEANT generated $100 \mathrm{GeV}$ electron and photon induced showers (each averaged over 50 showers) in ice with $0.611 \mathrm{MeV}$ threshold energy by GP given in Eq. (অ). The fitting parameters and the CL for the fits are given in Table 2. The fits with longitudinal profiles are plotted in Fig. 10. The critical energy in ice for the fits is 68.8 $\mathrm{MeV}$ as before. 


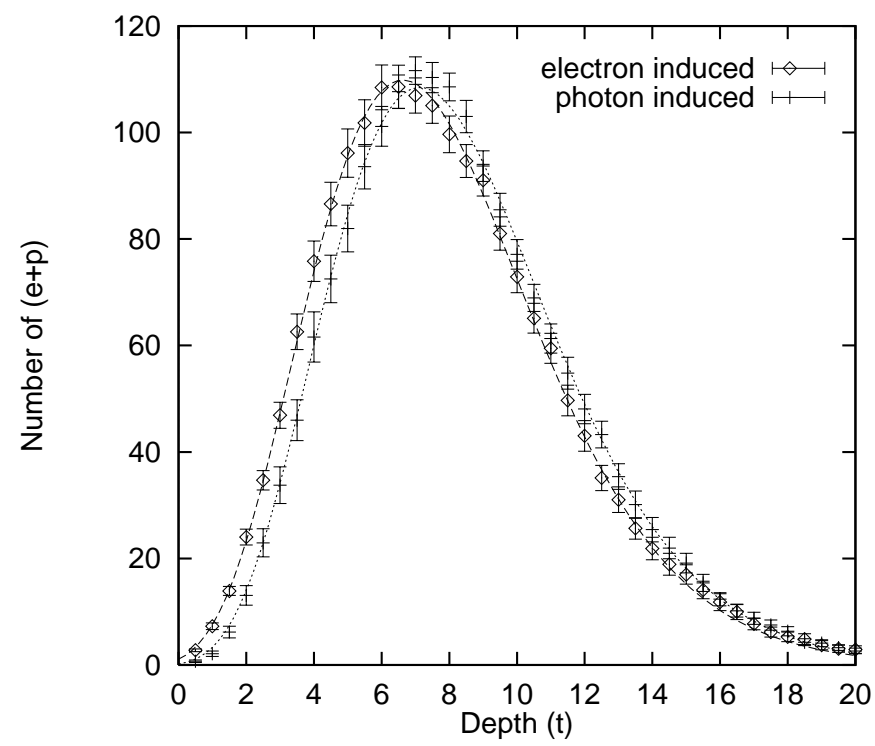

Figure 10: Greisen parametrization fits (smooth curves) to the longitudinal profiles of $100 \mathrm{GeV}$ electron and photon induced showers (each averaged over 50 showers) with $0.611 \mathrm{MeV}$ threshold energy from GEANT. The fitting parameters are given in Table 2. The critical energy for ice used in the fits is $68.8 \mathrm{MeV}$.

Table 2: Greisen parameters for GEANT showers in ice with $0.611 \mathrm{MeV}$ threshold and $E_{\mathrm{c}}=68.8 \mathrm{MeV}$.

\begin{tabular}{ccccc}
\hline \hline Primary & $E_{o}(\mathrm{GeV})$ & $A(E)$ & $a_{\pi, \gamma}$ & $\mathrm{CL}(\%)$ \\
\hline$\gamma$ & 100 & 0.65 & 0.16 & 85.6 \\
$e^{-}$ & 100 & 0.66 & 0.66 & 89.1 \\
\hline \hline
\end{tabular}

A three parameter fit to the GEANT generated $100 \mathrm{GeV}$ electron-induced shower in ice leaving the critical energy $\left(E_{\mathrm{c}}\right)$ as a free parameter along with $A(E)$ and $a_{\pi}$ gives $E_{\mathrm{c}}=58.3 \pm 3.1 \mathrm{MeV}$. This is within error bars of the critical energy $\left(E_{\mathrm{c}}=68.8 \pm 8.8\right.$ $\mathrm{MeV}$ ) found from Moliere radius calculation. The CL for this fit is $97.7 \%$.

A comparison of averages over 50 showers of $100 \mathrm{GeV}$ each from GEANT to the same from ZHS Monte Carlo (see Fig. 11) shows about a $25-35 \%$ discrepancy for the total number of particles at the shower max. The percentage discrepancy between the two simulations remains the same at higher energies.

The excess charge in the shower $(N(e-p) / N(e+p))$ is about $18 \%$ at the shower maximum, about twice Askaryan's original rough estimate [1].

Fig. 12 shows a longitudinal depth distribution of a much different kind. Unlike particles crossing planes transverse to the shower axis, here we look at the distribution of the complete count of excess electrons present in bins of one radiation length along the shower axis. The figure shows the distribution of excess electrons $(\mathrm{d} N(e-p) / \mathrm{d} t)$ in a $100 \mathrm{GeV}$ shower (averaged over 50 showers) with $0.611 \mathrm{MeV}$ total energy threshold. The distribution is broken down into different energy bins, showing that half of the excess 


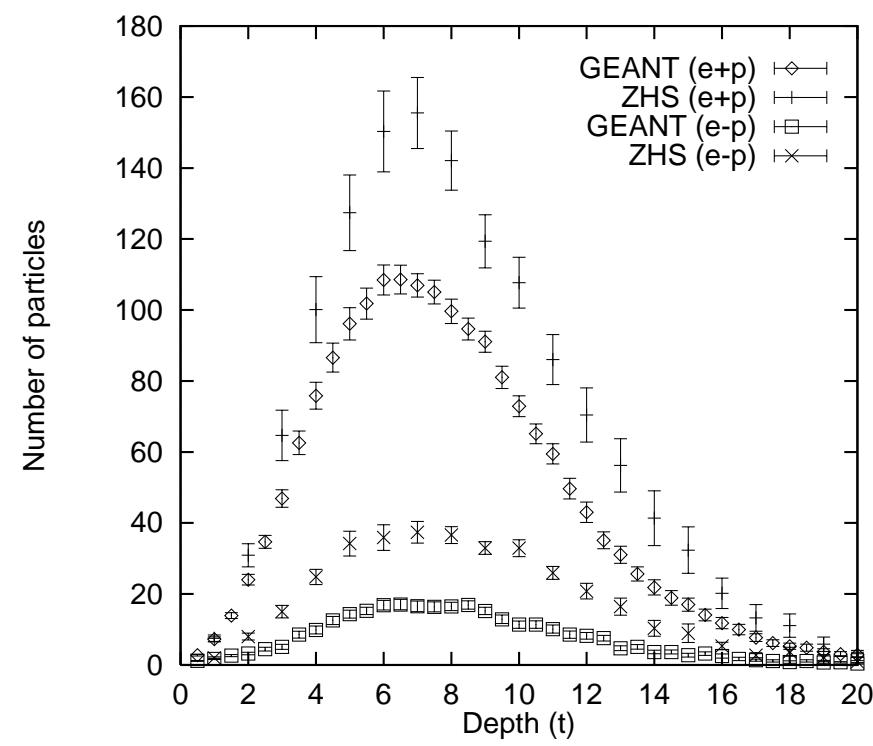

Figure 11: Comparison between shower profiles of a $100 \mathrm{GeV}$ shower (averaged over 50 showers in each case) from GEANT and from ZHS Monte Carlos. The error bars correspond to standard error, or $s / \sqrt{ } N$, where $s$ is the standard deviation and $N$ is the number of showers. The total energy threshold used is $0.611 \mathrm{MeV}$ in both cases. The difference in particle yield between the two Monte Carlos is $25-35 \%$ and remains the same at higher energy.

electrons have energy $5 \mathrm{MeV}$ or lower. This plot shows clearly the important point that the bulk of the particles at shower maximum have low energy and contribute significantly to the Cherenkov emission.

\subsection{1-D Model Study}

The comparisons in Figs. $8 \& 11$ prompt us to check the sensitivity of shower depth and particle number at maximum to the cross-sections assumed in a simulation, since ZHS and GEANT codes differ slightly in cross-sections for some processes (a few percent). To investigate this question in a setting that is completely under our control, we have written a one dimensional Monte Carlo that elaborates on Heitler's simple model to make it realistic. We include Compton scattering, positron annihilation, ionization loss, Moeller scattering, where interaction lengths are chosen from exponential distributions. The default values for the cross-sections are taken from GEANT. We examine the effect of changing crosssections of various processes. The total number of particles $(e+p$ and $\gamma)$ from the 1-D model scales linearly with shower energy and the position of the shower maximum scales logarithmically with energy as expected.

The effect of changing individual cross-sections (increasing and decreasing by $25 \%$ ) are listed in Table $3 \& 4$. We also list the total number of electrons and positrons $(N(e+p))$ at the maximum depth $\left(t_{\max }^{e+p}\right)$ and the total number of photons $(N(\gamma))$ at the maximum depth $\left(t_{\max }^{\gamma}\right)$ for the default values of the cross-sections (denoted by $\sigma_{\mathrm{o}}$ ). 


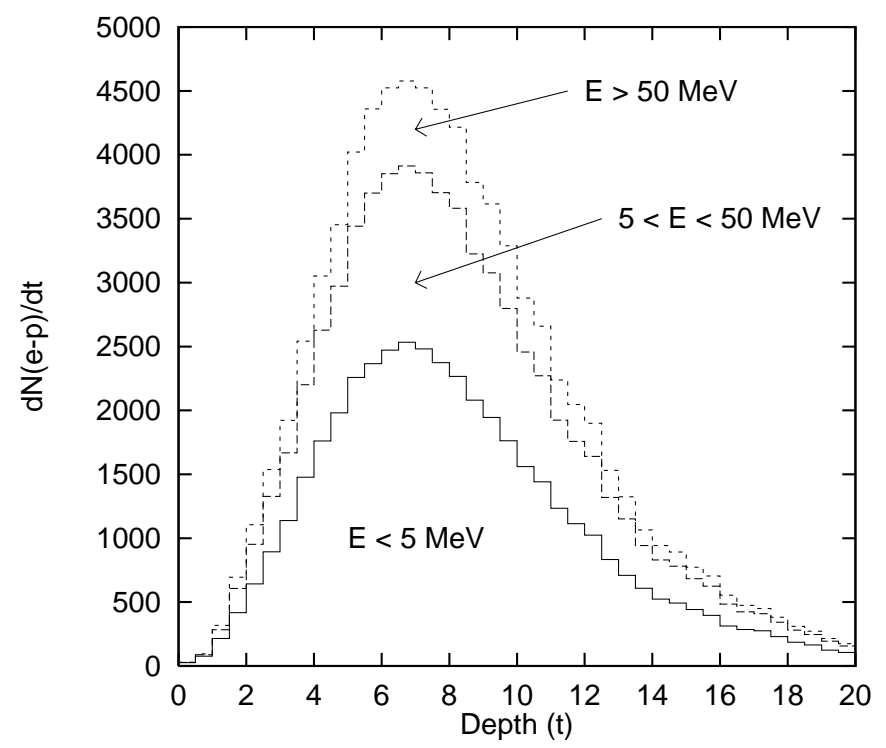

Figure 12: Depth distribution of excess charge $(\mathrm{d} N(e-p) / \mathrm{d} t)$ broken down into 3 different energy bins: $E<5 \mathrm{MeV}, 5<E<50 \mathrm{MeV}$ and $E>50 \mathrm{MeV}$. This figure shows that half of the excess electrons are created with energy below $5 \mathrm{MeV}$. We used a $100 \mathrm{GeV}$ shower (averaged over 50 showers) with $0.611 \mathrm{MeV}$ threshold to generate this plot.

Table 3: Results of increasing individual cross-section by $25 \%$ in the 1-D Monte Carlo. The default setting (GEANT) is denoted by $\sigma_{\mathrm{o}}$.

The cross-sections are changed one at a time from their default values.

\begin{tabular}{l|ccccccc}
\hline \hline Parameter & $\sigma_{\mathrm{o}}$ & Brem. & Pair & Comp. & $\delta$ & $\mathrm{d} E / \mathrm{d} x$ & Anni. \\
\hline$t_{\max }^{e+p}$ & 6.9 & 7.2 & 6.2 & 6.9 & 5.6 & 6.4 & 6.7 \\
$N(e+p)$ & 266 & 236 & 307 & 281 & 269 & 231 & 265 \\
$t_{\max }^{\gamma}$ & 6.9 & 7.0 & 5.9 & 6.4 & 6.4 & 6.7 & 6.4 \\
$N(\gamma)$ & 1418 & 1570 & 1495 & 1292 & 1396 & 1209 & 1416 \\
\hline \hline
\end{tabular}

Table 4: Results of decreasing individual cross-section by $25 \%$ in the 1-D Monte Carlo. The default setting (GEANT) is denoted by $\sigma_{\mathrm{o}}$. The cross-sections are changed one at a time from their default values.

\begin{tabular}{l|ccccccc}
\hline \hline Parameter & $\sigma_{\mathrm{o}}$ & Brem. & Pair & Comp. & $\delta$ & $\mathrm{d} E / \mathrm{d} x$ & Anni. \\
\hline$t_{\max }^{e+p}$ & 6.9 & 6.4 & 7.7 & 7.4 & 7.4 & 7.4 & 6.1 \\
$N(e+p)$ & 266 & 310 & 212 & 249 & 298 & 298 & 271 \\
$t_{\max }^{\gamma}$ & 6.9 & 6.7 & 8.2 & 7.4 & 6.9 & 6.9 & 6.4 \\
$N(\gamma)$ & 1418 & 1173 & 1259 & 1516 & 1544 & 1544 & 1399 \\
\hline \hline
\end{tabular}

The individual cross-sections are changed one at a time from their default values. The changes in the values of $t_{\max }$ and $N$ are noticeable, but the percentage changes are much smaller than the percentage changes in the cross-sections. For example, comparing the effect of increasing the bremsstrahlung cross section, listed in the column under "Brem." in Table 3 , the depth and number at maximum change by at most $4 \%$ and $15 \%$ respectively, 
compared to the default values, listed under " $\sigma_{\mathrm{o}}$, for a $25 \%$ change in the cross section. It seems that the shower features are not especially sensitive to precise values of an individual cross section. We have not made an exhaustive study of the effects of changing combinations of cross sections in all possible ways, but we have seen no indication that effects would be large without pathological distortions of the cross-sections.

\section{Shower Fluctuation}

Any determination of shower energy is intrinsically uncertain because of the fluctuations from shower to shower for the same injected energy. The fluctuations must be quantified compactly so an efficient and realistic energy uncertainty from this source can be assigned to detected showers. Fortunately, for UHE showers the huge numbers of particles and fractionally smaller fluctuations make this less problematic than for small showers. We summarize an approach to the fluctuation question in this section.

Conservation of total energy plays an important role in shower development. The shape of a shower strongly depends on the position and energy of the first hard bremsstrahlung the later the first hard bremsstrahlung event, the deeper the shower maximum is. However, for any shower, the primary energy (i.e., the total energy of the shower) qualitatively dictates the energy loss profile and the particle yield with depth.

It is common practice to describe the mean longitudinal profile of an electromagnetic shower by a Gamma distribution which is similar in shape to the Greisen parametrization. The Gamma distribution normalized to unity is given by

$$
f(t ; a, b)=b \frac{(b t)^{a-1} e^{-b t}}{\Gamma(a)} .
$$

However, Grindhammer et. al. 38 have shown that the fluctuations of the parameters $a$ and $b$ from an average profile do not necessarily follow the individual shower fluctuations. It is more reasonable to fit individual profiles by the Gamma distribution. One can then fit a 2-dimensional Gaussian distribution to the parameter set $\{a, b\}$ thus obtained and study shower fluctuation by studying these parameters. The correlation between these parameters $a$ and $b$ can be expressed as:

$$
\rho=\text { Covariance }(a, b) / \sigma_{a} \sigma_{b},
$$

where $\sigma_{a}$ and $\sigma_{b}$ are standard deviations of the parameters $a$ and $b$ respectively and

Covariance $(a, b)$ is the covariance matrix. The correlation $\rho$ is roughly independent of the energy of the shower.

\subsection{Fit to Simulations}

We have fitted the Gamma distribution Eq. (12) to 50 GEANT generated individual

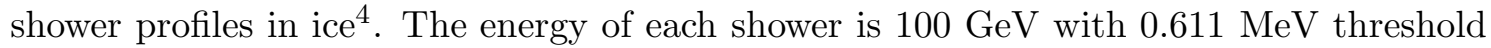
energy. The parameters $a$ and $b$ are extracted from the Gamma function fits. Two such fits to individual shower profiles are plotted in Fig. 13. 

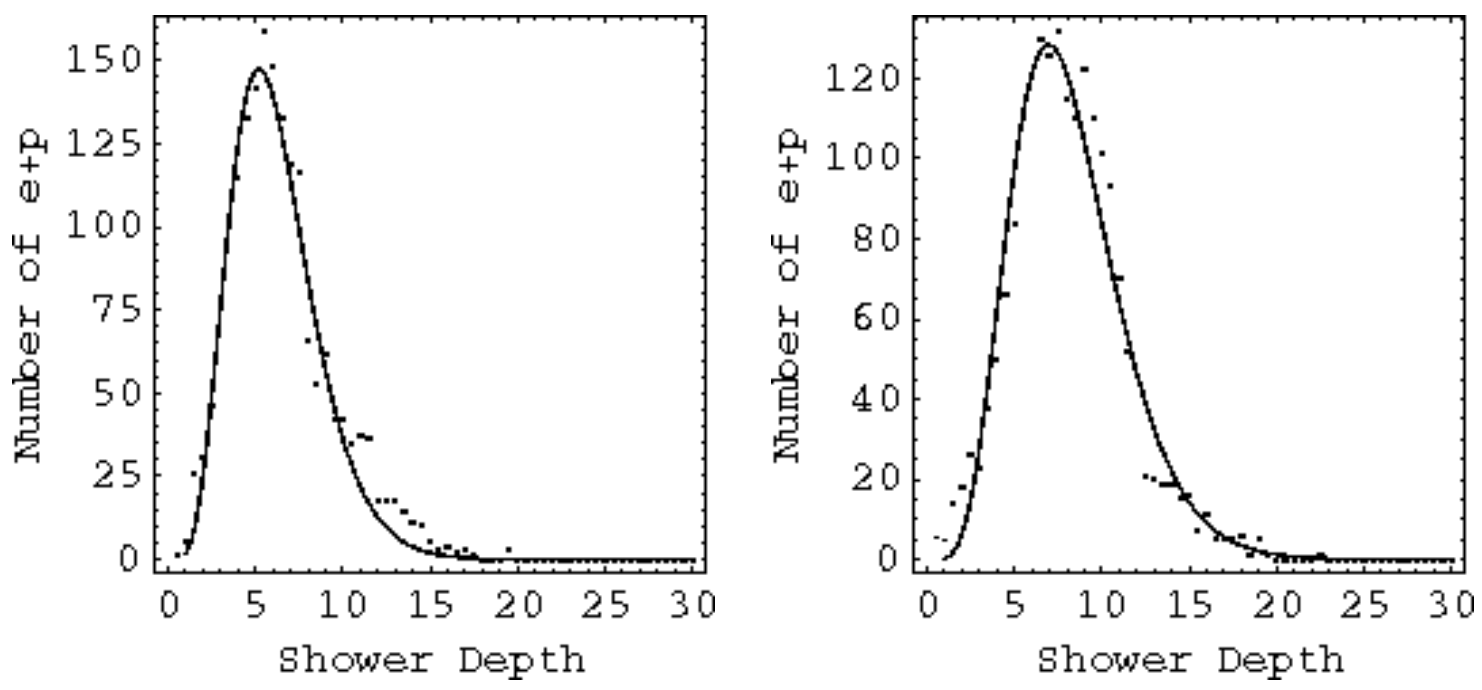

Figure 13: Individual shower profiles of $100 \mathrm{GeV}$ showers from GEANT with $0.611 \mathrm{MeV}$ threshold energy. The solid curves are Gamma function fits to the profiles. The parameters $a$ and $b$ in Eq. (12) are obtained from the fits.
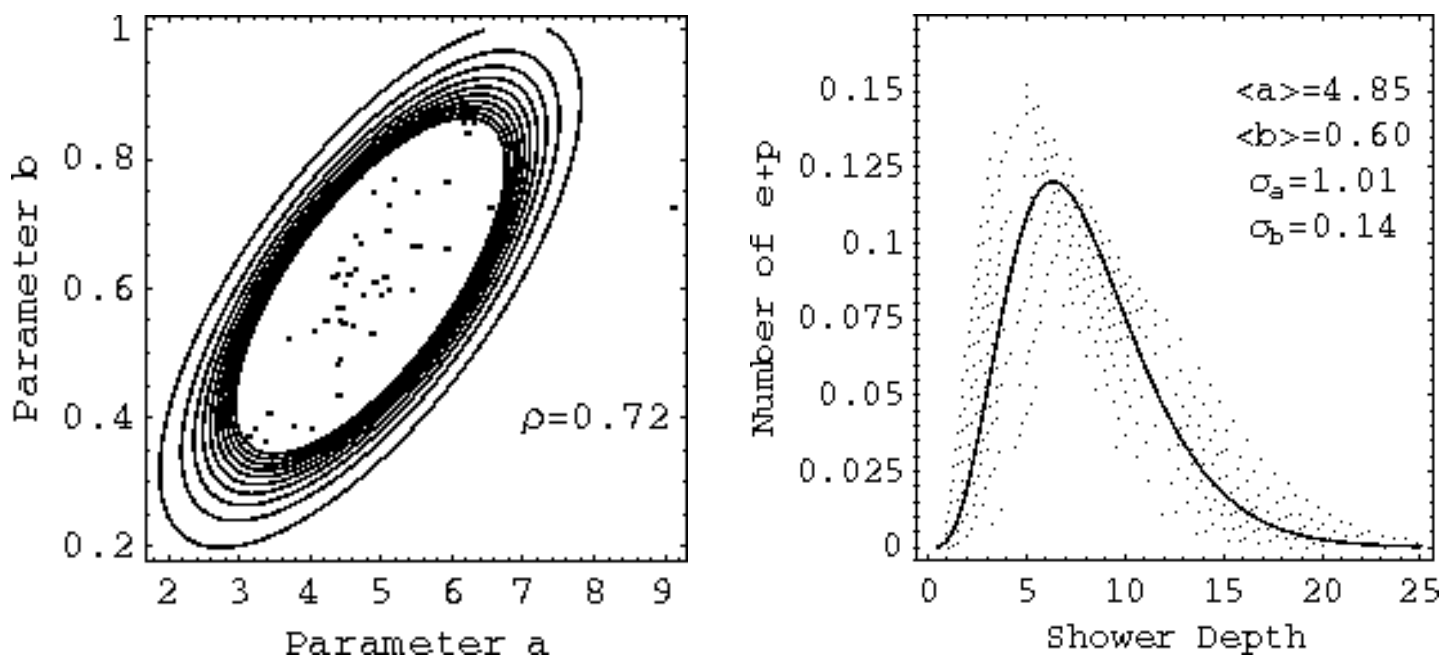

Figure 14: Scatter plot (left plot) of the parameters $a$ and $b$ from the Gamma distribution fit to 50 individual shower profiles each with energy $100 \mathrm{GeV}$ and $0.611 \mathrm{MeV}$ threshold. The contours are from a 2-dimensional Gaussian distribution with mean $(a, b)$ and $\left(\sigma_{a}, \sigma_{b}\right)$ obtained from the data set $a, b$ generated as described in the text. Shower fluctuations (right plot) due to variation of the parameters $a$ and $b$ within a standard deviation. The dark solid curve is the profile with mean values of $a$ and $b$. All particle numbers are normalized to 1 . 
Fig. 14a shows the scatter plot of the parameters $a$ and $b$ obtained by fitting 50 individual showers. We also show the contours of the 2-dimensional Gaussian distribution fit to the set of parameters $\{a, b\}$. This plot is similar in shape to the one obtained by Grindhammer using GEANT [38]. The correlation coefficient $\rho=0.72$ we found is close to 0.73 as found by Grindhammer et. al.

Fig. 14 b shows shower-to-shower fluctuations as we vary the parameters $a$ and $b$ within a standard deviation $\left(\sigma_{a}\right.$ and $\left.\sigma_{b}\right)$ of their mean values $(<a\rangle$ and $\left.\langle b\rangle\right)$. The dark solid curve corresponds to the shower with mean values of the parameters $a$ and $b$. As can be seen, the shower maximum can vary as much as $\sim 50 \%$. As remarked above, the variations become statistically less significant as the energy rises to the multi-PeV range, where UHE neutrino detectors such as RICE are sensitive. The uncertainties from fluctuations, though requiring study, are not expected to be a major part of the uncertainties in event locations and energies in any case.

\section{$5 \quad$ Electromagnetic Pulse Theory}

\section{Overview}

Given a current density, one calculates the electric field by straightforward application of Maxwell's equations. The most useful form of the electric field is the complex vector $\vec{E}_{\omega}(\vec{x})$, depending on three-dimensional coordinate $\vec{x}$ and angular frequency $\omega$. Before getting into the details of the radiation field calculation, we review its general features and several subtle points involving coherence, and near and far field limits.

The energy radiated per unit frequency interval per unit solid angle is proportional to $\left|E_{\omega}\right|^{2}$. In an asymptotically far field limit $R \rightarrow \infty$, the radiation field $E_{\omega} \propto 1 / R$, where $\mathrm{R}$ is the distance from the shower to the field point. Because the quantity $E_{\omega}$ has dimensions of mass in particle physics units and depends linearly on the track length $L$ (reviewed below), one expects the frequency dependence $E_{\omega} \propto L \omega / R$ on dimensional grounds. This linear dependence on $\omega$ breaks down at high frequencies, when wavelengths are smaller than the typical dimensions of the shower and create a coherence cutoff. The purpose of this section is to develop the concepts and formalism needed to address this and other issues.

The asymptotically far field is not a feature of Cherenkov physics as commonly presented in texts or applied in particle physics detectors. In particle physics applications, the track length is comparable to the distance to the observation point $R$. Textbook treatments take the limit that the track length is infinite, [39, 40, 41] in which case the electric field $E_{\omega} \sim \sqrt{\omega} / \sqrt{\rho}$, where $\rho$ is the cylindrical radial coordinate. In this situation, the radiation intensity is proportional to $\omega$, a familar feature of laboratory Cherenkov radiation. The cylindrical symmetry dictates this special frequency and radial dependence. The transition from cylindrical configuration to spherical configuration is associated with the terms "Fresnel and Fraunhoffer" zones. (Conditions of "far fields" $R \gg \lambda, \rho \gg \lambda$ are separate and assumed throughout; criteria separating the zones are given below.) The

\footnotetext{
${ }^{4}$ particle number $(e+p)$ normalized to 1 .
} 
conditions of the RICE experiment are such that spherical symmetry is a good approximation for the most distant events, $R \sim 1 \mathrm{~km}$, while Fresnel zone effects start to become noticeable for the nearest likely events, $R \sim 100 \mathrm{~m}$. Consequently both are reviewed.

There are two methods to calculate the electric field from the charged particles in a shower. One method calculates the electric field from each charged track and adds them by superposition. The other method parametrizes the shower's current and calculates electric fields analytically. Direct track-by-track calculation and superposition are presently limited to the Fraunhoffer zone. The result is simple, and depends only on the angle to the observation point. The analytic method works in either zone, and gives a compact way to take a few parameters characterizing the shower and calculate the field. Of course one must have a good parameterization for this to be a good approximation.

By using both the analytic and numerical methods, with their different strengths, we are able to characterize the coherence structure of showers in considerably more detail than previous work. The coherence extends to much higher frequencies than previously thought. This is a very important point, confirmed by two independent methods, and clears up a misconception that coherence should cut off at about $1 \mathrm{GHz}$.

\subsection{Fraunhoffer Limit}

The Fraunhoffer limit is appropriate to most of the RICE sensitive volume. It forms the basis for our numerical, track-by-track computation of the field produced by a shower at antenna sites remote from the shower. In this subsection, we derive the expression for the field produced by an individual track, which forms the basis for the calculation of the full field calculated from a whole shower. In the next subsection, we outline the method of parametrizing the shower as an effective current and calculating the field directly from that current.

The power at time $t$, position $\vec{x}$, radiated per unit solid angle by a moving electric charge is given, in Gaussian units, by [39]

$$
\frac{\mathrm{d} P(t)}{\mathrm{d} \Omega}=\frac{c}{4 \pi}\left|R \vec{E}(\vec{x}, t)_{\mathrm{ret}}\right|^{2},
$$

evaluated at the retarded time $t=t^{\prime}+R\left(t^{\prime}\right) / c$. (For now we calculate effects in vacuum; shortly we will supply the factors for the effects of a medium.) We use the Fourier transformed variables

$$
\begin{aligned}
R \vec{E}_{\omega} & =\frac{1}{\sqrt{2 \pi}} \int_{-\infty}^{\infty} R \vec{E}(t) e^{i \omega t} \mathrm{~d} t ; \\
R \vec{E}(t) & =\frac{1}{\sqrt{2 \pi}} \int_{-\infty}^{\infty} R \vec{E}_{\omega} e^{-i \omega t} \mathrm{~d} \omega .
\end{aligned}
$$

The energy radiated per unit frequency interval per solid angle is then given by

$$
\frac{\mathrm{d}^{2} I}{\mathrm{~d} \omega \mathrm{d} \Omega}=\frac{c}{4 \pi}\left|R E_{\omega}\right|^{2} .
$$

We will require the frequency dependence of the fields, so we work with the Fourier transformed fields below. 


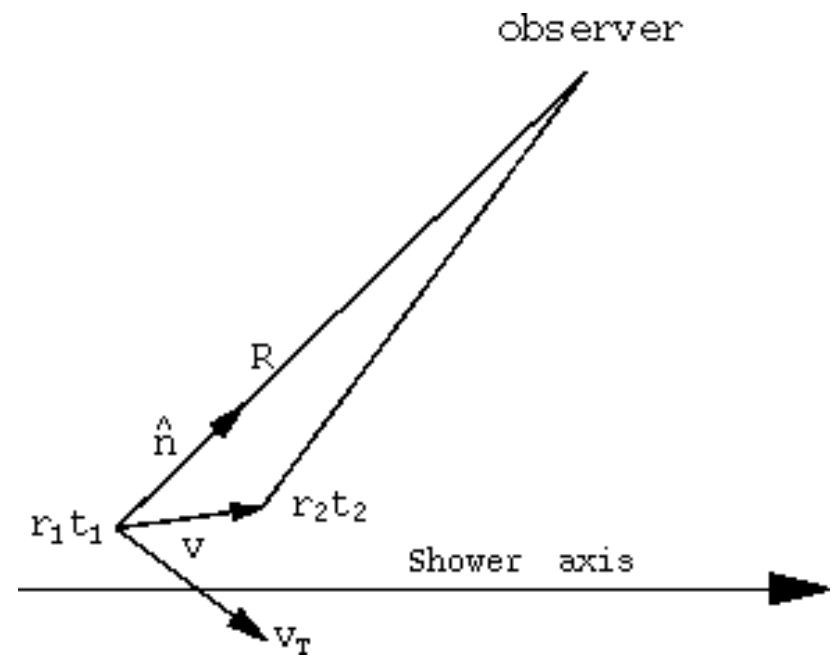

Figure 15: Geometry for calculating electromagnetic fields from a single track segment. $\left(r_{1}, t_{1}\right)$ and $\left(r_{2}, t_{2}\right)$ are the starting and ending position and time of the segment along which the particle moves with velocity $\vec{v}$.

The expression for the radiation field from a point source is conventionally defined as the electric field term linear in the acceleration $\dot{\vec{\beta}}$ :

$$
\vec{E}(\vec{x}, t)=\frac{q}{c}\left[\frac{\hat{n} \times\{(\hat{n}-\vec{\beta}) \times \dot{\vec{\beta}}\}}{(1-\vec{\beta} \cdot \hat{n})^{3} R}\right]_{\mathrm{ret}}
$$

where $\vec{\beta}$ is the velocity of the particle, $\hat{n}$ is the direction of the observer and $R$ is the distance from the track to the observation point (see Fig. 15). The factor $1 / R$ that accompanies the $\vec{\beta}$ factor is the other trademark of the radiation field. As is the case for the term that comes from the boosted Coulomb field, which has no explicit acceleration dependence, Eq. (18) is singular at the Cherenkov angle in a medium with real index of refraction greater than 1 .

Combining Eq. (15) and Eq. (18) we have

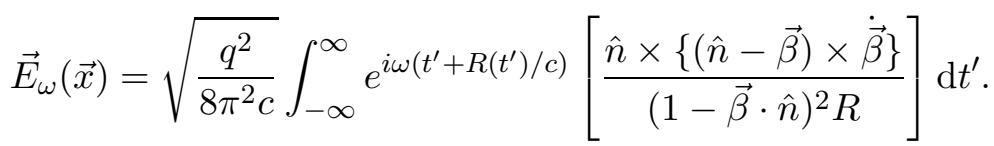

At distances large compared to the range of motion of the source, $\hat{n}$ is approximately constant and

$$
R\left(t^{\prime}\right) \approx|\vec{x}|-\hat{n} \cdot \vec{r}\left(t^{\prime}\right)
$$

This is the Fraunhoffer approximation: the error in the phase $\omega\left|\vec{x}-\vec{x}^{\prime}\right| / c$ must be kept small compared to $2 \pi$. Conditions for use of this approximation are discussed in the next 
subsection甲 (see Eq. (29)).

After integrating Eq. (19) by parts, and using the boundary conditions to set the end point contributions to zero, one finds [39]

$$
R \vec{E}_{\omega}(\vec{x}) \approx-i \omega \sqrt{\frac{q^{2}}{8 \pi^{2} c}} e^{i \omega R / c} \int_{-\infty}^{\infty} e^{i \omega(t-\hat{n} \cdot \vec{r} / c)}[\hat{n} \times(\hat{n} \times \vec{\beta})] \mathrm{d} t,
$$

where $R \equiv|\vec{x}|$. We will later apply this track-by-track expession to segments over which $\vec{\beta}$ is constant with time from $t_{1}<t<t_{2}$. On each segment $\vec{r}=\vec{r}_{1}+c \vec{\beta}\left(t-t_{1}\right)$. We insert the result into Eq. (21) and obtain

$$
R \vec{E}_{\omega}(\vec{x})=\frac{1}{\sqrt{2 \pi}}\left(\frac{q}{c}\right) e^{i k R} e^{i \omega\left(t_{1}-\hat{n} \cdot \vec{r}_{1} / c\right)} \vec{\beta}_{\perp} \frac{\left(e^{i \omega \delta t(1-\hat{n} \cdot \vec{\beta})}-1\right)}{1-\hat{n} \cdot \vec{\beta}}
$$

where $\hat{n} \times(\hat{n} \times \vec{\beta})=-\vec{\beta}_{\perp}$, and $\delta t=t_{2}-t_{1}$.

In a medium we replace $c \rightarrow c / n=c / \sqrt{\epsilon \mu}$, where $n=n(\omega)$ is the refractive index of the material and $\epsilon=\epsilon(\omega)$, the dielectric constant. We also replace $\vec{E} \rightarrow \vec{D}=\epsilon \vec{E}$ and $\vec{\beta} \rightarrow \vec{v} \frac{n}{c}=n \vec{\beta}$ everywhere, to get

$$
R \vec{E}_{\omega}(\vec{x})=\frac{1}{\sqrt{2 \pi}}\left(\frac{\mu_{r} q}{c^{2}}\right) e^{i k R} e^{i \omega\left(t_{1}-\frac{n}{c} \hat{n} \cdot \vec{r}_{1}\right)} \vec{v}_{\perp} \frac{\left(e^{i \omega \delta t(1-\hat{n} \cdot \vec{\beta} n)}-1\right)}{1-\hat{n} \cdot \vec{\beta} n}
$$

where $\mu_{r}$ is the relative permeability and $k=n \omega / c$.

The particle velocity $\vec{v}$ in the medium can be greater than that of light. The apparent singularity in Eq. (23) at $1-\hat{n} \cdot \vec{\beta} n=0$ defines the Cherenkov angle $\theta_{c}$ as $\cos \theta_{c} \equiv 1 / n \beta$. However there is no singularity, as seen by expanding the exponent. Close to the Cherenkov angle, Eq. (23) reduces to the form

$$
R \vec{E}_{\omega}(\vec{x})=\frac{i \omega}{\sqrt{2 \pi}}\left(\frac{\mu_{r} q}{c^{2}}\right) e^{i k R} e^{i \omega\left(t_{1}-\frac{n}{c} \hat{n} \cdot \vec{r}_{1}\right)} \vec{v}_{\perp} \delta t
$$

Eqs. (23 \& 24), used by ZHS without detailed derivation [9], are coded into the simulation to produce the field values for the tracks. Eq. (24) is explicitly linear in the track length $|\vec{v}| \delta t$, a feature we mentioned earlier.

Eqs. (23 \& 24) are incorporated into a track-by-track Monte Carlo simulation. The code uses the exact formula Eq. (23) unless the conditions are very close to the Cherenkov angle, posing a 0/0 numerical problem, in which case Eq. (24) is used.

Numerical summation of the electric field from all the tracks weighted by the proper charge automatically incorporates the features of coherence. As illustrated shortly, coherence produces a signal that peaks at the Cherenkov angle $\left(\theta_{c}\right)$, with a width away from the Cherenkov angle that shrinks with increasing frequency [9]. The coherence features are sufficiently intricate that we have devoted separate subsections to the topic.

\footnotetext{
${ }^{5}$ Analysis of the effects of keeping the next order in the expansion of the phase shows that significant deviations from the Fraunhoffer result appear at distances where the Fresnel zone sets in 42, as discussed from a general point of view below [43].
} 


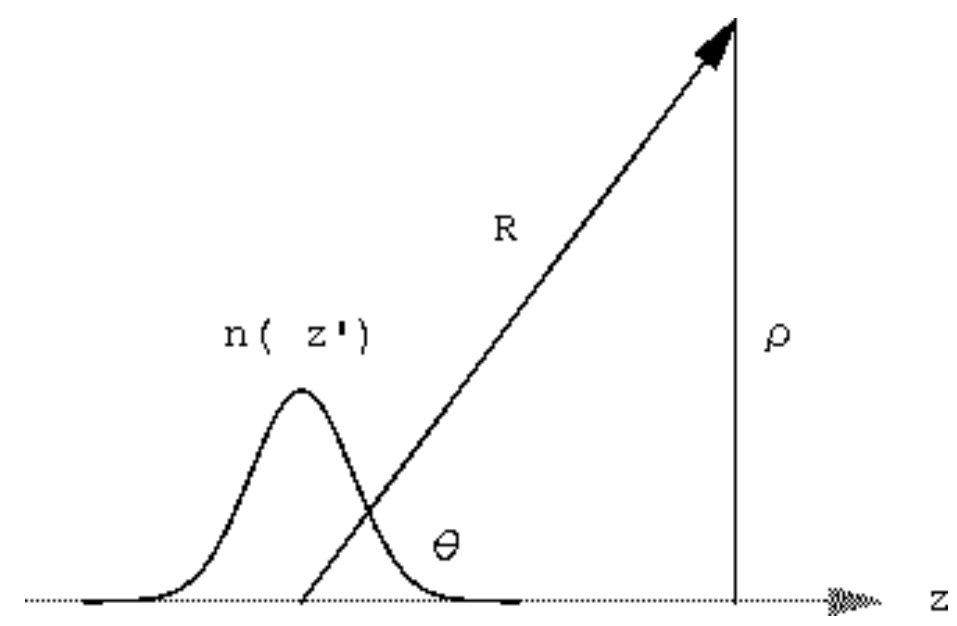

Figure 16: Shower parametrization is done by assuming the longitudinal excess charge development to be a Gaussian $n\left(z^{\prime}\right)$ moving along the shower axis (z-direction) with speed $v . \hat{n}$ is the direction of the observer, making an angle $\theta$ with the shower axis. $\rho$ is the transverse distance to the observer.

\subsection{Parametrization Method}

Charges of opposite sign which radiate coherently will give electric fields that cancel. It is the excess charge $(e-p)$ that determines the net field. Following Buniy and Ralston (BR) 433, one can then parameterize the excess (net) charge development in a shower and calculate the electric field due to the prescribed relativistic current. This is a flexible and compact approach that takes a few essential parameters from the shower and allows inspection of both the Fresnel and Fraunhoffer zones. We outline this method to keep our presentation self-contained.

The vector potential $\vec{A}$ in the Lorentz gauge (adapted to $\epsilon(\omega)$ and $\mu=1$ ) is given by

$$
\vec{A}_{\omega}(\vec{x})=\frac{4 \pi}{c \sqrt{2 \pi}} \int \mathrm{d}^{3} x^{\prime} \frac{e^{i k\left|\vec{x}-\vec{x}^{\prime}\right|}}{\left|\vec{x}-\vec{x}^{\prime}\right|} \int \mathrm{d} t^{\prime} e^{i \omega t^{\prime}} \vec{J}\left(t^{\prime} \vec{x}^{\prime}\right)
$$

The BR method parametrizes the excess charge development in the shower by a current along the shower axis $(z)$ as

$$
\vec{J}\left(t^{\prime}, \vec{x}^{\prime}\right)=\vec{v} n\left(z^{\prime}\right) f\left(z^{\prime}-v t^{\prime}, \vec{\rho}^{\prime}\right),
$$

where $\rho=\sqrt{x^{2}+y^{2}}$ is the radial distance from the shower axis, and $n\left(z^{\prime}\right)$ is the excess charge $(e-p)$ distribution, approximated by a Gaussian near the shower maximum (see Fig. 16), as

$$
n(z)=\frac{n_{\max }}{\sqrt{2 \pi}} e^{-z^{2} / 2 a^{2}} .
$$

This is analogous to Greisen and Rossi's parameterization [44, where $n_{\max }$ is the excess charge at the shower maximum, except that the excess (not total) charge $(e-p)$ is modeled in the BR case. The parameter a corresponds to the "longitudinal spread" of the shower 
near maximum and can be extracted by fitting a Gaussian to simulations of the excess charge profile of a shower.

The region of the shower over which the fields arrive nearly in phase at a distance $R(t)$ is called the coherence zone [43], and is given by

$$
\Delta z_{\mathrm{coh}}=\sqrt{\frac{R}{k \sin ^{2} \theta}},
$$

where $k=2 \pi / \lambda$ is the wave number and $\theta$ is the angle between $\hat{R}$ and the shower axis $(z)$. The value of the dimensionless ratio $\eta$,

$$
\eta=\left(\frac{a}{\Delta z_{\mathrm{coh}}}\right)^{2}=\frac{k a^{2}}{R} \sin ^{2} \theta
$$

determines how one should calculate the field. The ranges $\eta<1$ and $\eta>1$ correspond to the Fraunhoffer and Fresnel zones, respectively. In the case of $\eta \geq 1$, the series expansion of the phase, Eq. (20) fails, and the exact phase must be kept. As noted earlier, both the Fraunhoffer and Fresnel zones are far-field problems in the sense that $k R \gg 1$ is assumed in both cases.

For $\omega \sim \mathrm{GHz}$ the Fraunhoffer approximation is appropriate for $R>100 \mathrm{~m}$ distances typical of the RICE experiment. However, for a test beam experiment, where the interplay between different length scales is important, the Buniy-Ralston method to calculate the field in the Fresnel zone is required.

\subsection{Radiated Power and The Form Factor}

We return to the ansatz Eq. (26), which describes a current with transverse distribution independent of the longitudinal evolution. This is a reasonable assumption after the first few radiation lengths of the shower development. We make the specific ansatz

$$
\vec{J}\left(t^{\prime}, \vec{x}^{\prime}\right)=\hat{e}_{z} q v n\left(z^{\prime}\right) g\left(\vec{\rho}^{\prime}\right) \delta\left(z^{\prime}-v t^{\prime}\right)
$$

Note that the transverse extent of showers is small compared to the longitudinal extent. The phase and the denominator in Eq. (25) can be safely expanded in cylindrical coordinates as

$$
\begin{aligned}
\left|\vec{x}-\vec{x}^{\prime}\right| & =\sqrt{\left(z-z^{\prime}\right)^{2}+\rho^{2}-2 \vec{\rho} \cdot \vec{\rho}^{\prime}+\rho^{\prime 2}} \\
& \approx R\left(z, z^{\prime}\right)\left(1-\frac{\rho \rho^{\prime}}{R^{2}} \cos \phi^{\prime}\right)+\mathcal{O}\left(\frac{R^{\prime 2}}{R^{2}}\right)
\end{aligned}
$$

and

$$
\frac{1}{\left|\vec{x}-\vec{x}^{\prime}\right|} \approx \frac{1}{R}
$$

\footnotetext{
${ }^{6}$ Shown in detail earlier (Eqs. (23 \& 24)).
} 
where $R\left(z, z^{\prime}\right)=\sqrt{\left(z-z^{\prime}\right)^{2}+\rho^{2}}$ and $R^{\prime}=\sqrt{z^{\prime 2}+\rho^{\prime 2}}$. This expansion of the transverse variables avoids the Fraunhoffer approximation, and leads to a "factored" expression for the field:

$$
R \vec{E}_{\omega}(\vec{x}) \approx I F(\omega)
$$

where $I$ is an integral characteristic of the longitudinal shower history, and $F(\omega)$ is called the form factor. The integral $I$ can be evaluated in the saddle-point approximation, as detailed elsewhere [43], or evaluated by Monte Carlo. This feature of factorization to express the field using a form factor works in both the Fraunhoffer and Fresnel zones.

In the Fraunhoffer zone, there is a further, and very remarkable, simplification. Keeping the term linear in $z^{\prime}$, and dropping terms of order $R^{\prime 2} / R^{2}$ in the phase expansion, the electric field at the Cherenkov angle $\left(\theta_{\mathrm{c}}\right)$ is

$$
\begin{aligned}
R \vec{E}_{\omega}(\vec{x})= & q 2 \sqrt{2 \pi} i e^{i k R}\left[\hat{e}_{z}\left(\frac{\omega}{c^{2}}-\frac{k \cos \theta_{\mathrm{c}}}{\epsilon v}\right)-\hat{e}_{\rho} \frac{k \sin \theta_{\mathrm{c}}}{\epsilon v}\right] \\
& \times \int \mathrm{d} z^{\prime} n\left(z^{\prime}\right) \int \mathrm{d} \rho^{\prime} \rho^{\prime} g\left(\rho^{\prime}\right) \int \mathrm{d} \phi^{\prime} e^{-i \frac{n \omega}{c} \rho^{\prime} \sin \theta_{\mathrm{c}} \cos \phi^{\prime}}
\end{aligned}
$$

It is remarkable that all terms in the phase exponent depending on $z^{\prime}$ have vanished. This means that radiation from the shower is coherent over the entire length of the shower in the Fraunhoffer approximation: the "coherence zone" is limited only by the track length . In obtaining these results, the Cherenkov condition $\cos \theta_{\mathrm{c}}=c / n v=1 / n \beta$ and the approximations: $\cos \theta_{\mathrm{c}}=z / R, \sin \theta_{\mathrm{c}}=\rho / R$ (see Fig. 16) and $\rho^{\prime} / R \ll 1$ were used.

Inspecting the transverse integral, we see that it alone contributes to the form factor $F(\omega)$, which is given by

$$
\begin{aligned}
F(\omega) & =\int \mathrm{d}^{2} x_{\perp}^{\prime} e^{-i \vec{k}_{\perp} \cdot \vec{x}_{\perp}^{\prime}} g\left(\vec{x}_{\perp}^{\prime}\right), \\
& =\int_{0}^{\infty} \mathrm{d} \rho^{\prime} \rho^{\prime} g\left(\rho^{\prime}\right) \int_{0}^{2 \pi} \mathrm{d} \phi^{\prime} e^{-i \frac{n \omega}{c} \rho^{\prime} \sin \theta_{\mathrm{c}} \cos \phi^{\prime}}, \\
& =2 \pi \int_{0}^{\infty} \mathrm{d} \rho^{\prime} \rho^{\prime} g\left(\rho^{\prime}\right) J_{\mathrm{o}}\left(\frac{n \omega}{c} \rho^{\prime} \sin \theta_{\mathrm{c}}\right) .
\end{aligned}
$$

Here $J_{\mathrm{o}}$ is the Bessel function of order unity. Just as in particle physics usage, $F(\omega)$ is the Fourier transform of the (transverse) excess charge distribution.

The preceding analysis leads to a convenient expression for the electric field at the Cherenkov angle in the Fraunhoffer approximation, which apart from some normalization factors is given by

$$
\left|R \vec{E}_{\omega}(\vec{x})\right|=q 2 \sqrt{2 \pi} I(a) \frac{\omega \sin \theta_{\mathrm{c}}}{c^{2}}|F(\omega)| .
$$

The radiated power at the Cherenkov angle $\left(\theta_{\mathrm{c}}\right)$ depends on the form factor only. As noted earlier, the same form factor can be used in both the Fraunhoffer and Fresnel limits, so that laboratory information about the form factor can be used directly. The form factor is the central topic of subsequent sections discussing the coherence.

\footnotetext{
${ }^{7}$ The importance of the coherence zone is well known in the Landau-Pomeranchuk-Migdal [14, 15, 17] effect, where the lengthening coherence zone suppresses bremsstrahlung. The Cherenkov condition causes the coherence zone to expand to equal the entire track length.
} 


\section{Electric Field Pulse Calculation}

In this section we carry out the computational outline just developed. The vector nature of superposition is taken into account. The analytic construction of the form factor, developed earlier, is adapted to the numerical calculation, so that two independent methods can be compared. We observe using the simulation data that coherence extends far above the frequency regime anticipated from simple estimates using characteristic scales such as the Moliere radius.

\subsection{Vector Superposition}

To calculate the electric pulse from a GEANT generated shower, we summed the contributions to the electric field in Eqs. (23 \& 24) from all charged track segments using full 3-dimensional geometry. We denote the observation point by a unit vector, $\hat{n}=(\sin \theta \cos \phi, \sin \theta \sin \phi, \cos \theta)$. The starting time $t_{1}$ of each track segment is obtained from GEANT output. The time interval $\delta t$ for each track-segment is calculated for a particle of total energy $E$ (from GEANT output) traveling at constant speed $\beta$ throughout the track segment. Slowing of low energy particles is thus taken care of approximately. The electrical field has an azimuthal symmetry which will be investigated later.

The electric field is a complex vector quantity. As such there are two questions of coherence, one to do with phase and one to do with the vector nature of the field. The vector nature of the field depends on the term $\hat{n} \times(\hat{n} \times \vec{v})=-\vec{v}_{\perp}$ in Eqs. (23 \& 24). This construction picks out the component of the velocity which is perpendicular to the direction of the observer. Since the track segments vary in direction, we add the electric field contributions as vectors which allow any cancellation that may occur. The electric field amplitude is then proportional to the track length transverse to the direction to the observer $\left(|\vec{v}|_{\perp} \delta t\right)$. On the Cherenkov cone, one then expects that the field generated by the entire shower is proportional to the track length projected along the shower axis times the sine of the Cherenkov angle. The projected track length thus accounts for the vector nature of the electric field for tracks that go in different directions, and serves as an important diagnostic of the whole procedure.

However, the projected track length says nothing in itself about the conditions for phase coherence, which is the topic of the next subsection.

\subsection{Phase Coherence}

The numerically generated phases of the fields, track-by-track, are determined by the complex exponentials in Eq. (23). We call the phase angles $\omega\left(t_{1}-\frac{n}{c} \hat{n} \cdot \vec{r}_{1}\right)$ and $\omega \delta t(1-$ $\hat{n} \cdot \vec{\beta} n)$ the translational phase (TP) and the Cherenkov phase (CP) respectively. The translational phase is kinematic, a consequence of translational invariance, and depends on the beginning of each track segment. The Cherenkov phase vanishes at the Cherenkov angle, which a point of stationary phase and dominates the emission. Two or more track segments will contribute in phase if the observer lies on the Cherenkov cone, and the beginnings of the tracks do not destructively interfere. 

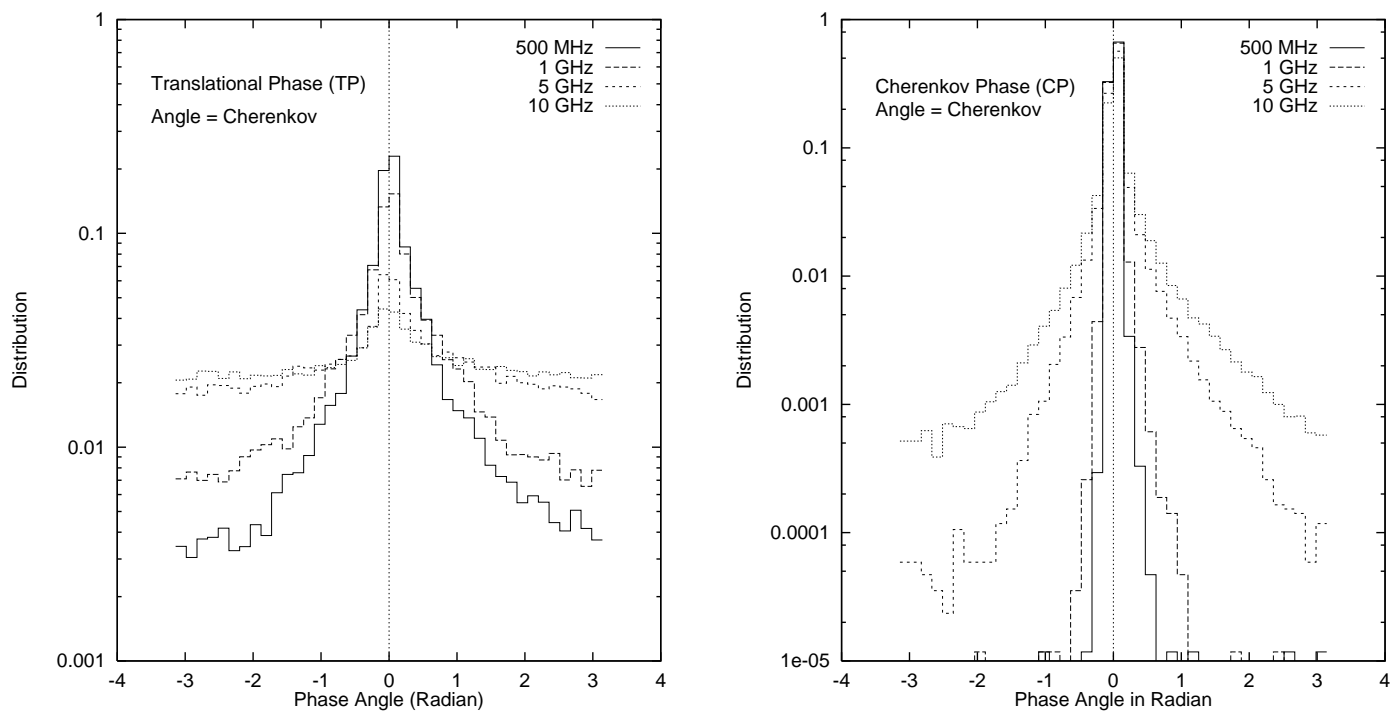

Figure 17: Distributions of the translational phase (TP) and the Cherenkov phase $(\mathrm{CP})$ at the Cherenkov angle $\left(\theta=\theta_{\mathrm{c}}\right)$ for a single shower of energy $100 \mathrm{GeV}$ and threshold of $0.611 \mathrm{MeV}$. The TP (left plot) shows strong coherence (sharp peak) at low frequencies. A slight positive enhancement of the TP can be interpreted as particle positions slightly lagging the light cone because $\beta<1$. The $\mathrm{CP}$ (right plot), in comparison, remains strongly peaked at all frequencies, which underscores the argument that the phase $(\mathrm{CP})$ varies a little over the whole track.

We studied the distribution of the TP and $\mathrm{CP}$ at the Cherenkov angle $\left(\theta \approx 55.8^{\circ}\right)$ and at an angle $\left(\theta=40^{\circ}\right)$ off the Cherenkov angle (Figs. 17 \& 18). We used a single $100 \mathrm{GeV}$ shower with $0.611 \mathrm{MeV}$ total energy threshold to make the phase distribution plots. We studied the distribution at frequencies: $10 \mathrm{GHz}, 5 \mathrm{GHz}, 1 \mathrm{GHz}$ and $500 \mathrm{MHz}$.

The distribution of the TP (Fig. 17) shows a strong peak at low frequency, indicating coherent phase emission from track segments at the Cherenkov angle. At high frequencies, the distribution tends to become flat, with random phases indicating a loss of coherence. A net positive phase indicates the particle positions slightly lagging behind the light cone due to their speed $\beta$ slightly less than 1. The flat distribution of the TP in Fig. 18, on the other hand, clearly indicates random phases coming from track segments at angles off the Cherenkov cone.

The distribution of the $\mathrm{CP}$ remains qualitatively the same both at the Cherenkov angle (Fig. 17) and at angles off the Cherenkov angle (Fig. 18). It is also apparent that frequency dependence of the $\mathrm{CP}$ is very weak.

The preceding discussion makes clear that phase coherence is dominated by the TP. Finally, the distribution of TP and CP variables are substantially uncorrelated (see Figs. $19 \&$ 20). These features indicate a valid factorization of the electric field, leading to an independent motivation for the Ansatz Eq. (30) and consequent appearance of the form factor. We discuss this factorization in the following subsection. 

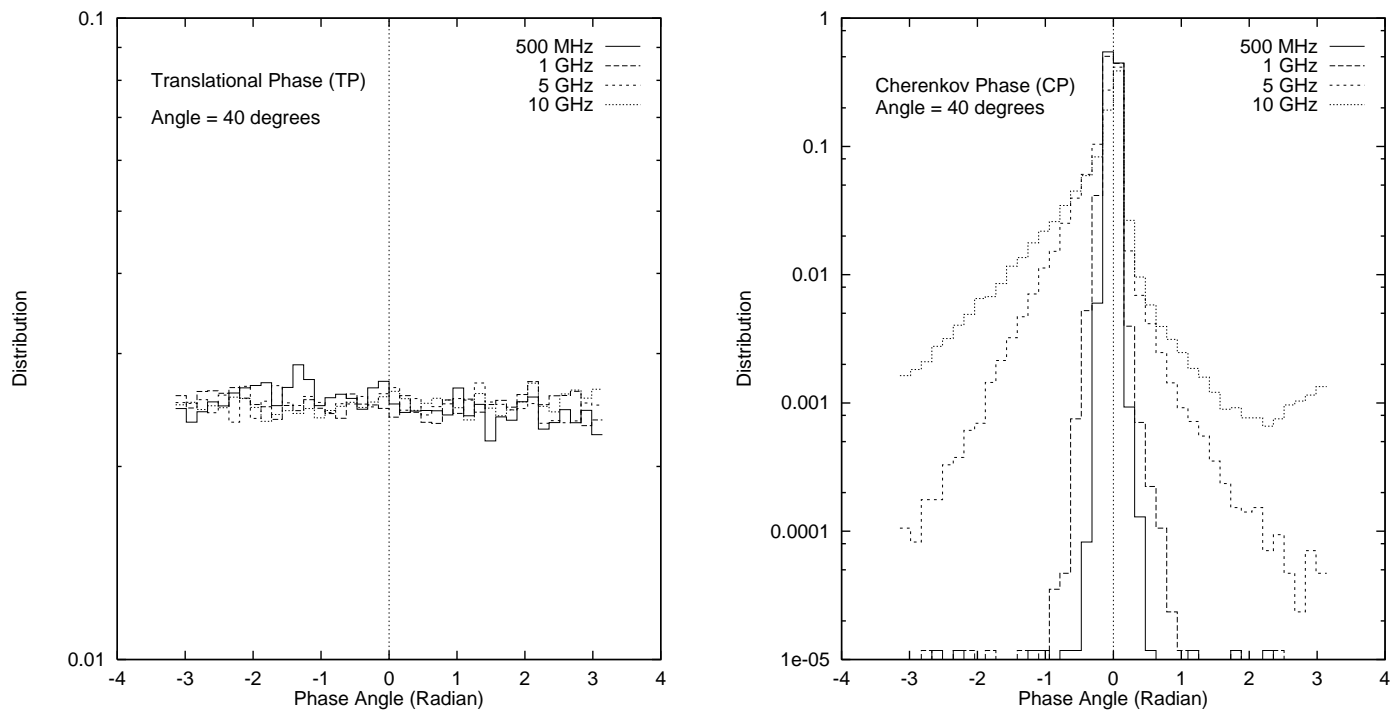

Figure 18: Distributions of the TP and the $\mathrm{CP}$ at an angle $\left(\theta=40^{\circ}\right)$ off the Cherenkov cone for a single shower of energy $100 \mathrm{GeV}$ with total energy threshold of $0.611 \mathrm{MeV}$. The flat distributions of the TP (left plot) at all frequencies indicates the randomness of phases coming from randomly located track segments. The CP distribution (right plot), is qualitatively the same as the case on Cherenkov angle. Taken together, these features indicate that phase coherence is dominated by the TP.
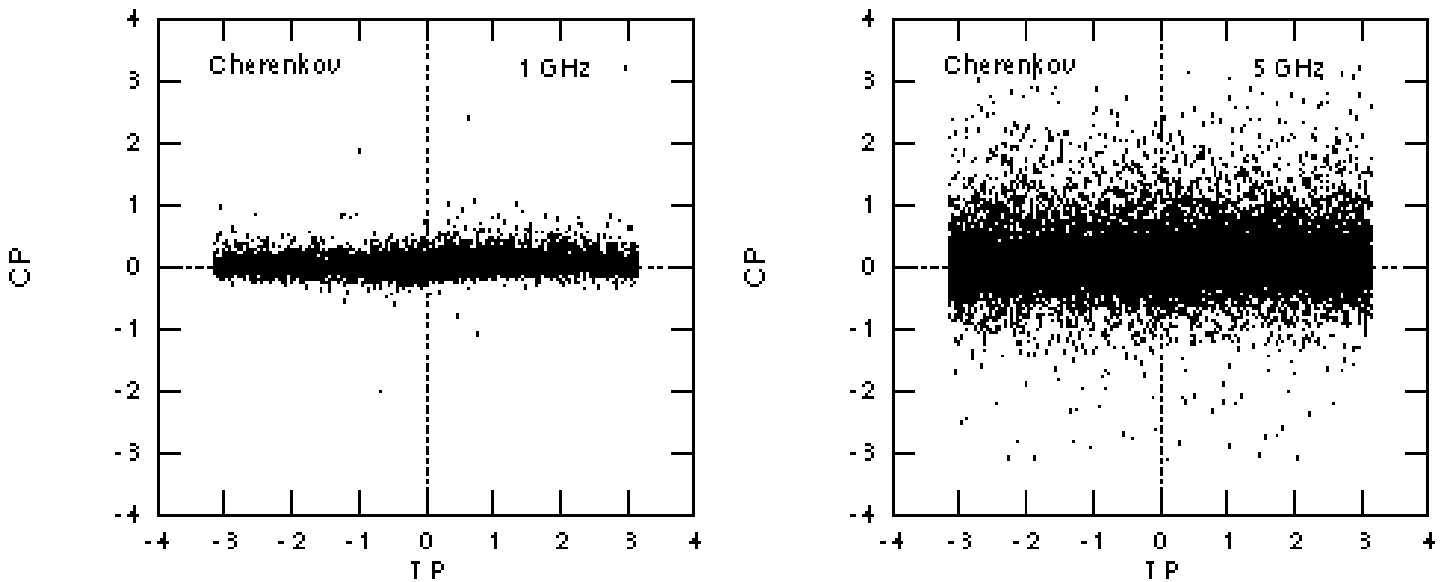

Figure 19: Scatter plots of the translational phase (TP) and the Cherenkov phase (CP) at the Cherenkov angles $\left(\theta_{\mathrm{c}}\right)$ and at frequencies: $1 \mathrm{GHz}$ (left plot) and $5 \mathrm{GHz}$ (right plot). A correlation between the two variables would appear as a slanted line, or similar feature. The plots demonstrate that the translational and Cherenkov phases are substantially uncorrelated. 

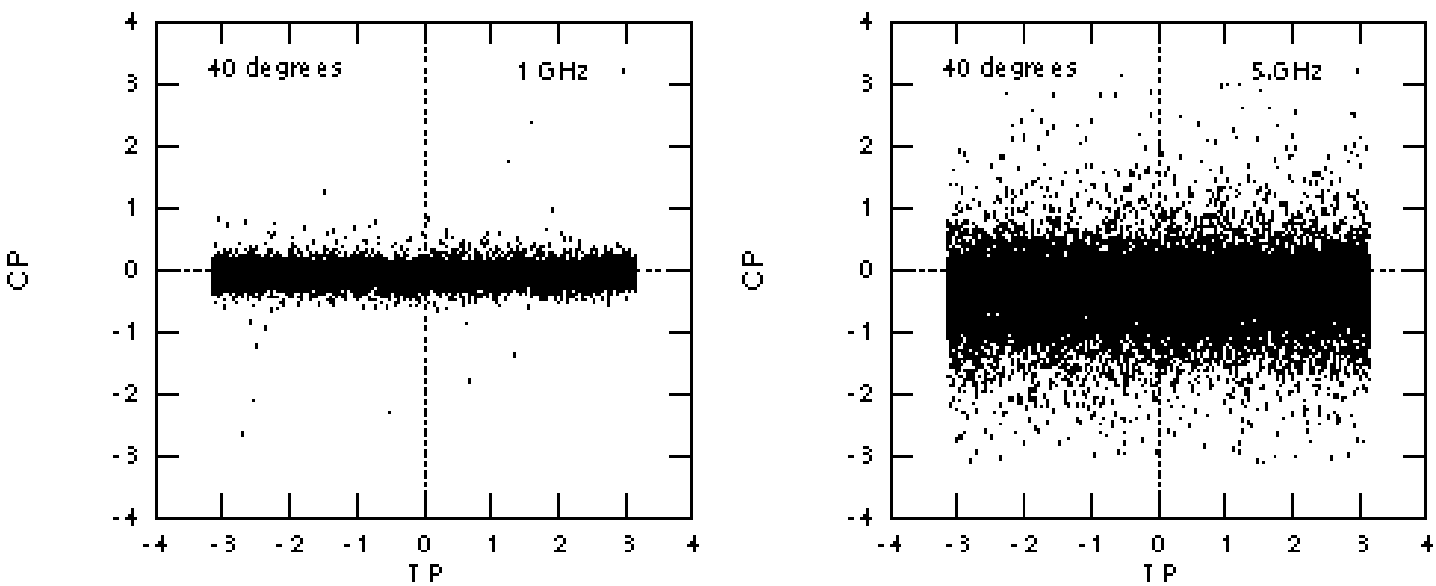

Figure 20: Same as Fig. 19 at angle $\left(\theta=40^{\circ}\right)$.

\subsection{Factorization of the Electric Field, and the Discrete Form Factor}

The electric field from all the shower particles can be factorized because the TP and CP phases are uncorrelated, as shown above. We write the field in factored form as

$$
E_{\omega}^{\mathrm{tot}} \propto \sum_{j} e^{i \phi_{j}^{\mathrm{TP}}}\left(e^{i \phi_{j}^{\mathrm{CP}}}-1\right) \sim \sum_{j} e^{i \phi_{j}^{\mathrm{TP}}} \sum_{j}\left(e^{i \phi_{j}^{\mathrm{CP}}}-1\right),
$$

where the sums are over all track segments. The factorization takes a rather simple form at the Cherenkov angle. The electric field close to the Cherenkov angle in Eq. (24) can be written approximately for a single track as

$$
R \vec{E}_{\omega}(\vec{x})=\frac{i \omega}{\sqrt{2 \pi}}\left(\frac{q}{c^{2}}\right) \vec{v}_{\perp} \delta t e^{i k R} e^{i \omega z_{1}\left(\frac{1}{v}-\frac{n}{c} \cos \theta\right)} e^{-i \frac{n \omega}{c} \hat{n} \cdot \vec{r}_{1 \perp}}
$$

The approximation $t_{1} \simeq z_{1} / v$ has been assumed in writing Eq. (38), which is well supported by the TP phase plot at the Cherenkov angle shown above. The phase angle $\omega z_{1}(1 / v-n \cos \theta / c)$ vanishes for the Cherenkov condition $\left(\cos \theta_{\mathrm{c}}=c / n v\right)$ and we have full coherence along the $z$-direction (shower axis) as observed earlier.

We now write the factorization in Eq. (37) for the total electric field at the Cherenkov angle from all the charged particles in the shower as

$$
R \vec{E}_{\omega}^{\text {tot }}(\vec{x})=\frac{i \omega}{\sqrt{2 \pi}}\left(\frac{q}{c^{2}}\right) e^{i k R} \sum_{j} s^{j}\left(\vec{v}_{\perp}\right)^{j} \delta t^{j} e^{-i \frac{n \omega}{c} \hat{n} \cdot\left(\vec{r}_{1 \perp}\right)^{j}}
$$

where $s^{j}= \pm 1$ for positrons and electrons respectively. The total electric field in Eq. (39) is thus proportional to the total track length $\left(\sum_{j} s^{j}\left(\vec{v}_{\perp}\right)^{j} \delta t^{j}\right)$ transverse to the direction of the observer at any frequency $\omega$. This track length is the projected $(e-p)$ track length times $\sin \theta_{c}$, as described earlier.

The coherent electric field emission at different frequencies now depends on the Monte Carlo "discrete form factor" $F(\omega)_{\mathrm{MC}}$, given by

$$
F(\omega)_{\mathrm{MC}}=\sum_{j} s^{j} e^{-i \frac{n \omega}{c} \hat{n} \cdot\left(\vec{r}_{1}\right)^{j}}=\sum_{j} s^{j} e^{-i \frac{n \omega}{c} x_{1}^{j} \sin \theta_{\mathrm{c}}} .
$$



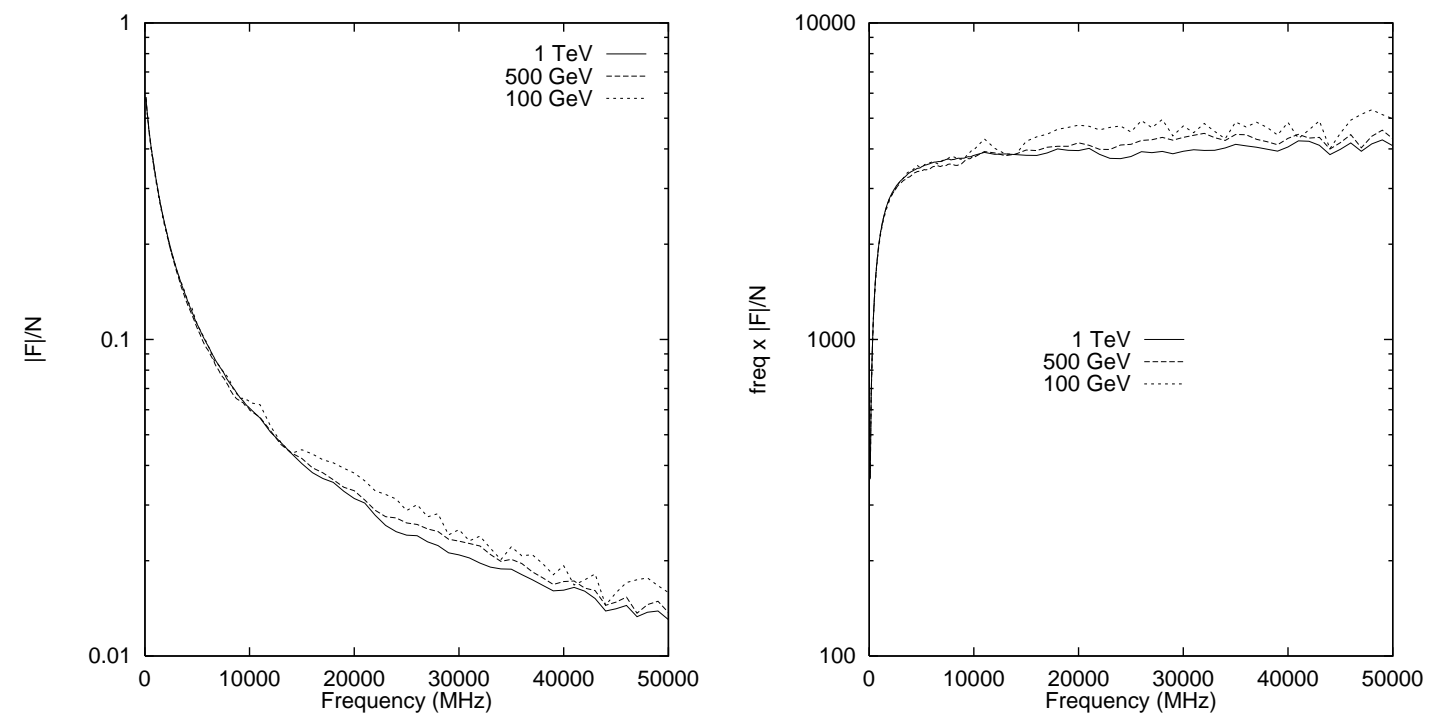

Figure 21: Absolute value of the form factor $(|F(\omega)|)$ (left plot) and the frequency spectrum $(\omega|F(\omega)|)$ (right plot) plots for $1 \mathrm{TeV}, 500 \mathrm{GeV}$ and $100 \mathrm{GeV}$ showers. The form factor has been calculated using Eq. (40), where we naively sum over all the shower particles along the $z$-axis (shower axis). The curves are normalized by dividing by the particle count $N$.

Note that the observation point is in the $x-z$ plane.

\subsection{The Discrete Form Factor and the Frequency Spectrum}

We calculated the discrete form factor in Eq. (40) for $1 \mathrm{TeV}, 500 \mathrm{GeV}$ and $100 \mathrm{GeV}$ showers. A factor of $\omega$ times the form factor is proportional to the electric field amplitude.

First, we naively carried out the sum in Eq. (40) for all the shower particles along the whole shower axis $(z)$ as suggested by the Cherenkov condition in the Eq. (38). The absolute value of the form factor and the frequency spectrum are plotted in Fig. 21. It shows an extended region of coherence, with $\mid$ Form factor $\mid \sim N / \omega$, where $N$ is the number of shower particles. This naive estimate is somewhat misleading because the Cherenkov condition is not satisfied for the whole shower axis. The $\mathrm{CP}$ also becomes flat at very high frequencies, as does the TP at the Cherenkov angle (see Fig. 17a). A further complication is statistical fluctuations, which become large where the form factor is small.

For a better understanding of the form factor and the frequency spectrum, we now turn to the analytic method.

\subsection{The Analytic Form Factor and the Frequency Spectrum}

The analytic form factor as defined in Eq. (35) is the Fourier transform of the snapshot of the charge distribution. It is a good approximation to assume that most of the electric field contribution comes from the particles at the shower maximum [43].

To calculate the analytic form factor, we then determined the transverse distribution of the particles within half a radiation length on both sides of the shower maximum for a 


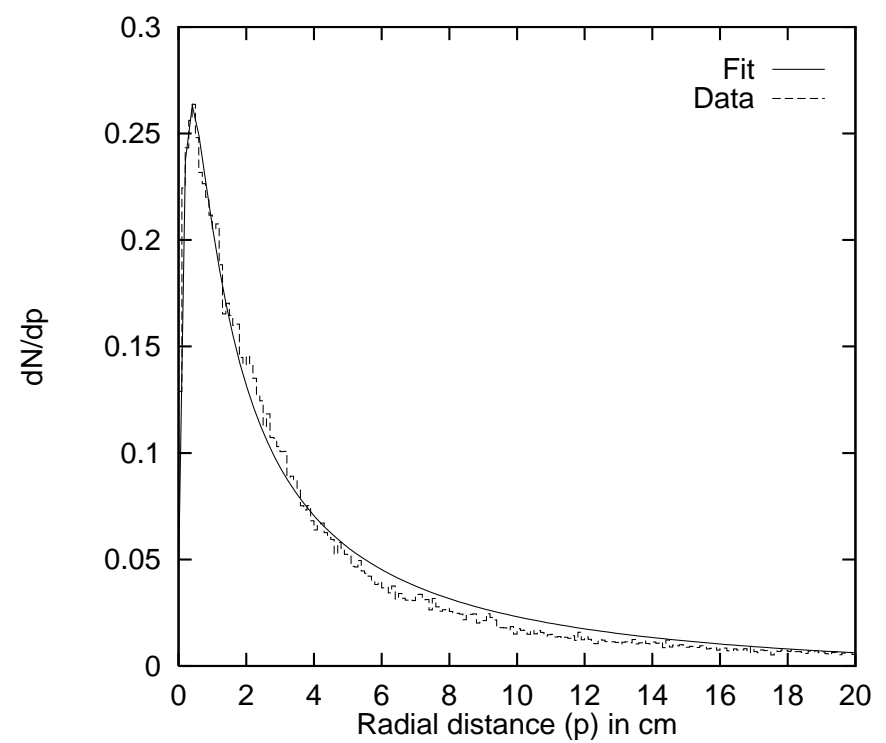

Figure 22: Radial distributions $\mathrm{d} N / \mathrm{d} \rho$ of the excess $(e-p)$ electrons within half a radiation length on both sides of the shower maximum for a $100 \mathrm{GeV}$ shower (averaged over 50 showers). The solid curve is a Pade(1,3) fit to the excess charge distribution.

$100 \mathrm{GeV}$ shower (averaged over 50 showers). We found both the distribution of the total particles $\mathrm{d} N(e+p) / \mathrm{d} \rho$ and the distribution of the excess electrons $\mathrm{d} N(e-p) / \mathrm{d} \rho$. Here, $\rho$ is the cylindrical radial distance from the shower axis. The excess charge distribution $(\mathrm{d} N(e-p) / \mathrm{d} \rho)$ is plotted in Fig. 22 .

We observed that the radial distribution falls approximately like $1 / \rho^{2}$ for large $\rho$, and peaks at a remarkably small value of $0.5 \mathrm{~cm}$. One way (by no means complete) to understand these results recalls the multiple scattering of an electron by a static Coulomb field. For a screened Coulomb field, the modified Rutherford scattering cross section is given by [39]:

$$
\frac{\mathrm{d} \sigma_{s}}{\mathrm{~d} Q}=8 \pi\left(\frac{Z e^{2}}{\beta c}\right)^{2} \frac{Q}{\left(Q^{2}+Q_{s}^{2}\right)^{2}}
$$

where $Q$ is the momentum transfer and $Z$ is the atomic number of the scatterer; $Q_{s}=$ $\left(Z^{1 / 3} / 192\right) m c$ is the momentum transfer associated with the screening radius; $m$ is the mass of the electron. For elastic scattering in the ultra-relativistic limit, $Q^{2}=2 p^{2}(1-$ $\cos \theta) \approx p^{2} \theta^{2}$, where $\mathrm{p}$ is the electron momentum. The cross-section formula in Eq. (41) now takes the form:

$$
\frac{\mathrm{d} \sigma_{s}}{\mathrm{~d} \theta}=8 \pi\left(\frac{Z e^{2}}{\beta c}\right)^{2} \frac{p^{2} \theta}{\left(p^{2} \theta^{2}+Q_{s}^{2}\right)^{2}} .
$$

The average deflection angle, $\langle\theta\rangle \approx \rho / \Delta z$. We can get a crude estimate of the transverse distribution $\mathrm{d} N / \mathrm{d} \rho$ from Eq. (42) as:

$$
\frac{\mathrm{d} N}{\mathrm{~d} \rho}=8 \pi\left(\frac{Z e^{2}}{\beta c}\right)^{2}\left(\frac{n \Delta z^{3}}{p^{2}}\right) \frac{\rho}{\left(\rho^{2}+\rho_{\mathrm{o}}^{2}\right)^{2}}
$$


where $n$ is the number density related to $N$ by the formula $N=n \sigma \Delta z$. The peak of this distribution is given by

$$
\rho_{\mathrm{o}}=\frac{Q_{s} \Delta z}{p} .
$$

We evaluate this with the average energy of the particles taken to be approximately equal to the critical energy $\left(E_{\mathrm{c}}\right)$ at the shower maximum. The peak of the distribution is then $\rho_{\mathrm{o}}=0.5 \mathrm{~cm}$ for ice $\left(Z=7.2, \Delta z=39 \mathrm{~cm}\right.$ and $\left.p \sim E_{\mathrm{c}}=70 \mathrm{MeV}\right)$. The excellent agreement may be fortuitous, but gives some confidence that the Monte Carlo excess charge distribution, which also includes numerous atomic collision, pair production and Compton scattering processes, has a simple physical origin.

We made a fit to the excess charge distribution with a Pade $(1,3)$ approximation of the form $\mathrm{d} N / \mathrm{d} \rho=f(\rho)=n(\rho-a) /\left(1+b \rho+c \rho^{2}+d \rho^{3}\right)$ (see Fig. 22). The fitting parameters are: $n=2.15, a=0.01, b=4.73, c=6.96, d=0.33$. The fit is good up to $\rho \sim 20 \mathrm{~cm}$. The choice of the Pade fit was made to preserve the $\rho^{1}$ geometric zero in $\mathrm{d} N / \mathrm{d} \rho$ at the origin, and the $1 / \rho^{2}$ asymptotic behavior.

Finally, we calculated the analytic form factor using Eq. (35) as:

$$
F(\omega)=2 \pi \int_{0}^{\infty} \mathrm{d} \rho f(\rho) J_{\mathrm{o}}\left(\frac{n \omega}{c} \rho \sin \theta_{\mathrm{c}}\right) .
$$

For the high frequency end it was necessary to use a convergence procedure to modulate the Bessel transform. We compare the analytic prediction to the discrete form factor of Eq. (40) at the shower maximum for $100 \mathrm{GeV}, 500 \mathrm{GeV}$ and $1 \mathrm{TeV}$ showers. The comparison is shown in Fig. 23. For reference we also plotted the analytic and the discrete frequency spectra, which are $\omega$ times the absolute value of the form factor. The agreement between the two methods is good up to $10 \mathrm{GHz}$ for all energies and up to $50 \mathrm{GHz}$ for $1 \mathrm{TeV}$.

\subsection{Direct Calculation: Monte Carlo Field Spectrum}

The frequency spectrum of the electric field calculated using Eqs. (23 \& 24) at the Cherenkov angle and an angle off the Cherenkov angle are plotted in Fig. 24a. We calculated the spectrum for $1 \mathrm{TeV}, 500 \mathrm{GeV}$ and $100 \mathrm{GeV}$ showers (each averaged over many showers). The electric field amplitude rises linearly at low frequency: this is the linear dependence on $\omega$ due to dimensional analysis. The coherent behavior at the Cherenkov angle and the incoherent behavior at a widely separated angle $\left(40^{\circ}\right)$ are clear. The frequency spectrum of the electric field calculated from a $100 \mathrm{GeV}$ GEANT generated shower is compared to the same from the ZHS code in Fig. 24 b.

From the previous analysis, the frequency dependence of the electric field amplitude is expected to be $\omega|F(\omega)|$. We compare $\omega|F(\omega)|$ to $\left|E_{\omega}\right|$ generated by the Monte Carlo in Fig. 25. The electric field amplitude at the Cherenkov angle is proportional to the projected $(e-p)$ track length $\times \sin \theta_{\mathrm{c}}$ as shown in Eq. (39). We used the total projected $(e-p)$ track length value of 70 meter (as we found before) for a $100 \mathrm{GeV}$ shower as a pre-factor to normalize the analytic form factor. It is clear from the plot that the analytic form factor and simple dimensional considerations explain the electric field amplitude rather well up through 5-15 GHz range. The coherence persists up to frequencies as high as $50 \mathrm{GHz}$. We 

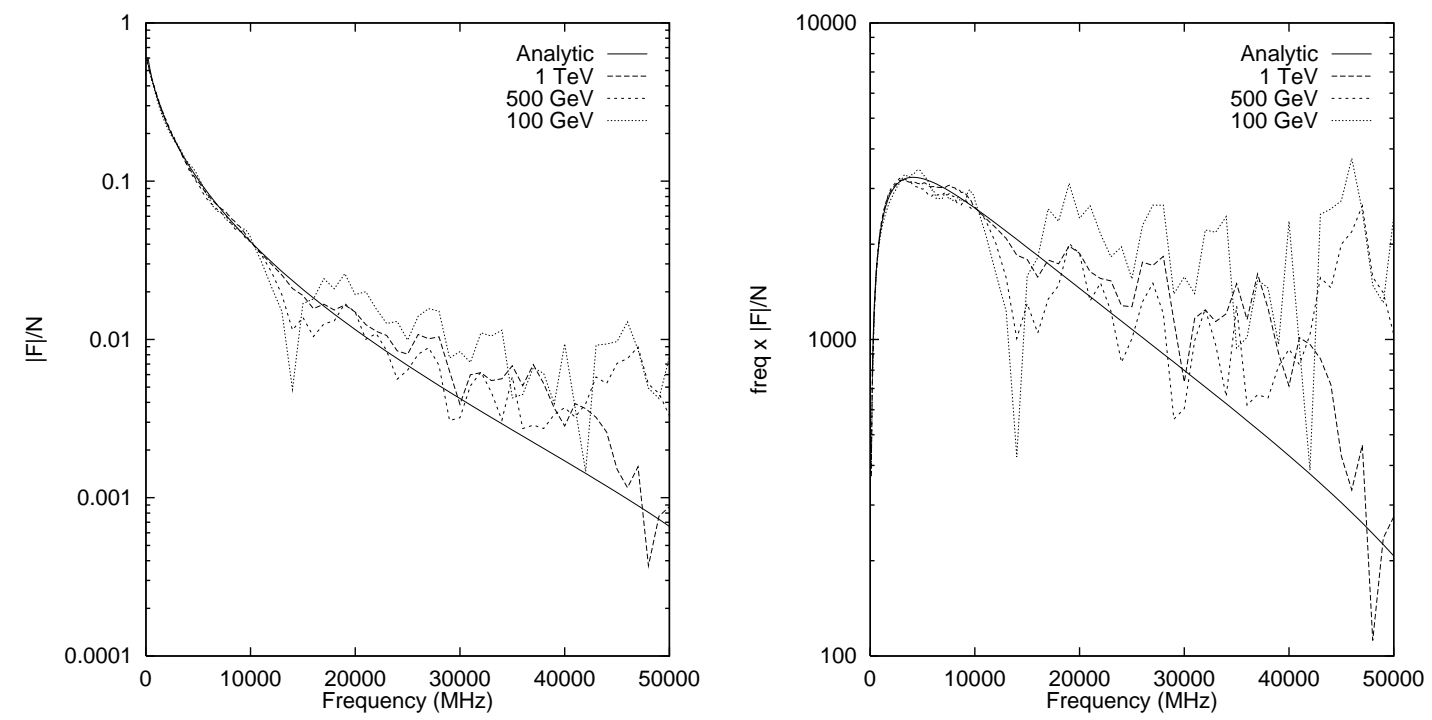

Figure 23: Absolute value of the form factor $|F(\omega)|$ (left) and the frequency spectrum $\omega|F(\omega)|$ (right) plotted versus frequency for $1 \mathrm{TeV}, 500 \mathrm{GeV}$ and $100 \mathrm{GeV}$ showers. The discrete form factor has been calculated using Eq. (40), where we sum over all the particles within a radiation length at the shower maximum. The analytic form factor has been calculated from the Fourier transform to a fit to the excess charge distribution (see Fig. 22). Both curves are normalized by dividing by the particle count $N$ Note that the analytic curve tends to be lower than the numerical calculation at high frequency.

notice that the agreement between the direct Monte Carlo calculation of the frequency spectrum and the analytic frequency spectrum gets better with increasing shower energy. We believe that the analysis captures the important physics of the processes, and validates the results of the Monte Carlo.

\subsection{Related Issues}

Electrons and positrons undergo Coulomb scattering while traversing through the medium. The particle track is therefore not a straight line from the point of its creation to the point where it falls below threshold. The track contains many kinks due to elastic Coulomb scattering. GEANT gives a detailed output of particle tracks which contain these kinks as stated earlier; we calculate the electric field from step-tracks. The Monte Carlo developed by ZHS, on the other hand used the straight tracks from the start points to the end points, with timing correction, to calculate field [9] to simplify their calculation.

We studied the effect of these kinks and also the effect of taking all tracks along the shower axis (see Fig. 26). We found little difference between the cases when we included (the usual case) and not included the kinks. This is a result of the extended coherence zone along the shower axis at the Cherenkov angle. When we calculated the electric field taking only the components parallel to the shower axis, we found that the field increases almost linearly with frequency, as expected from a single charge.

For practical purposes, particles are removed from the Monte Carlo simulation once 

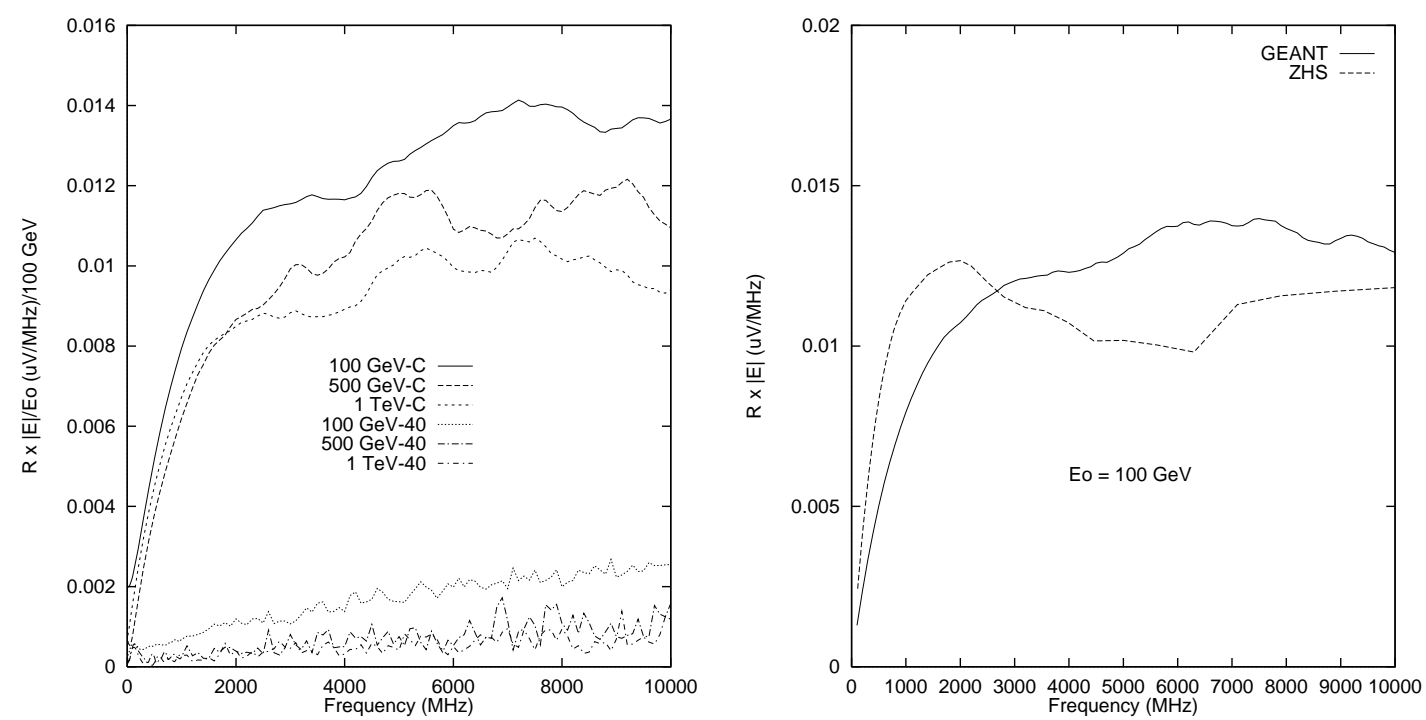

Figure 24: Direct Monte Carlo calculation of the frequency spectrum of the electric field magnitude for $1 \mathrm{TeV}, 500 \mathrm{GeV}$ and $100 \mathrm{GeV}$ showers (left). The calculation is done using Eqs. (23 \& 24) at the Cherenkov angle and at $40^{\circ}$ angle. The coherent behavior at the Cherenkov angle and the incoherent behavior at the $40^{\circ}$ viewing angle are clear. The frequency spectrum at the Cherenkov angle for a $100 \mathrm{GeV}$ shower is also compared to the same calculated using ZHS code (right). All thresholds are $0.611 \mathrm{MeV}$ and the averages are done with 50 showers in case of 100 $\mathrm{GeV}, 20$ showers in case of $500 \mathrm{GeV}$ and 10 showers in case of $1 \mathrm{TeV}$.

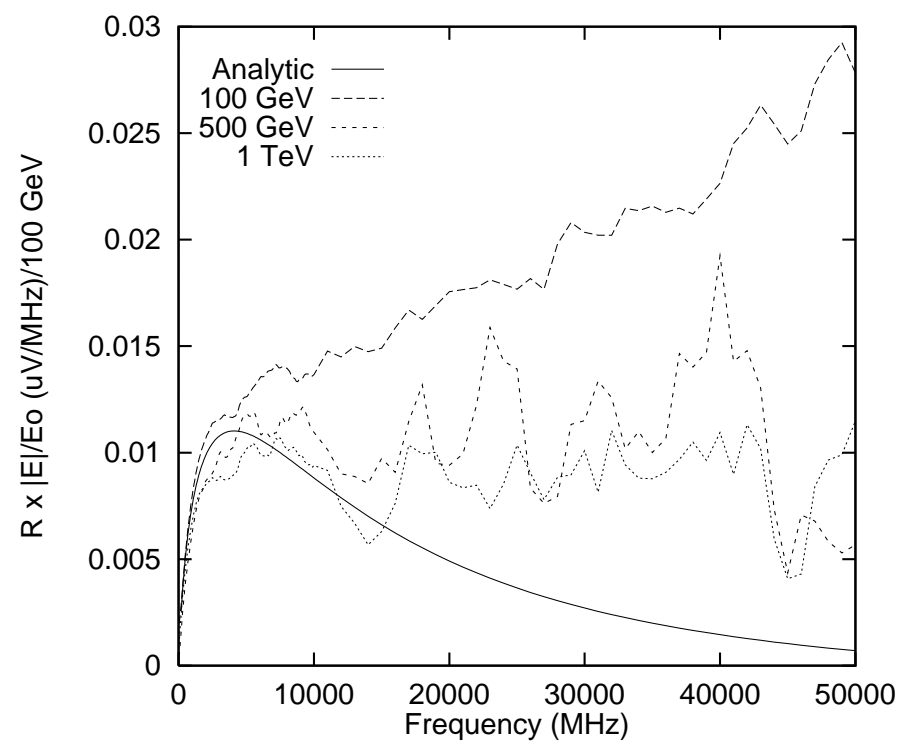

Figure 25: Comparison between the frequency spectra of the electric field magnitude at the Cherenkov angle from direct Monte Carlo calculation and from analytic calculation. The analytic calculation is done using Eq. (40) with the total projected track length of $70 \mathrm{~m}$ for a $100 \mathrm{GeV}$ shower as found before. 


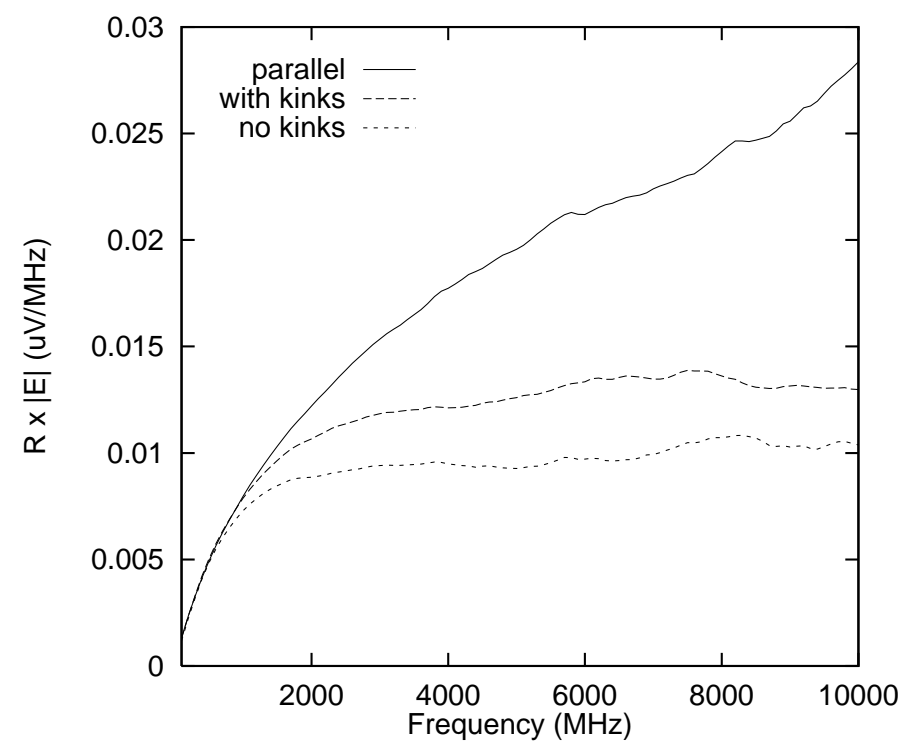

Figure 26: Frequency spectrum of the electric field magnitude at the Cherenkov angle for a $100 \mathrm{GeV}$ shower (averaged over 50 showers). Our study shows that the electric field calculated using particle tracks with and without kinks make little difference. The electric field magnitude, on the other hand increases almost linearly with frequency like a single charge when we took the component of the tracks parallel to the shower axis only.

they fall below certain threshold energy (0.611 MeV in our case). While physical particles do not suddenly stop moving, simulated particles in the Monte Carlo, have potential emission of stopping radiation. Even though high energy particles are almost parallel to the shower axis, the low energy particles close to threshold are isotropic. The stopping radiation (which is forward) from all these isotropic particles in general could cause a problem if they are aligned with the observer on the Cherenkov cone.

Our study shows (see Fig. 27) that there is indeed an isotropic component in the shower below energy $0.85 \mathrm{MeV}$. However, they do not contribute to the radiation at the Cherenkov angle $\left(\theta_{\mathrm{c}} \approx 55.8^{\circ}\right)$. Particles with energy $1 \mathrm{MeV}$, for example, Cherenkov radiates at an angle $49^{\circ}$.

\subsection{Angular Pulse Distribution}

The angular distribution of the electric field amplitude peaks at the Cherenkov angle $\left(\theta_{\mathrm{c}} \approx 55.8^{\circ}\right)$. Fig. 28 shows the $1 \mathrm{GHz}$ pulse from a $100 \mathrm{GeV}$ shower (averaged over 50 showers) with $0.611 \mathrm{MeV}$ total energy threshold at 4 different azimuthal angles $\phi=$ $90^{\circ}, 180^{\circ}, 270^{\circ}$ and $360^{\circ}$. The pulse shows very little dependence on $\phi$ which corresponds to the approximately symmetric distribution of particles about the shower axis as stated earlier. We have also plotted the electric field amplitude from the ZHS Monte Carlo for a $100 \mathrm{GeV}$ shower (averaged over 50 showers) at $1 \mathrm{GHz}$ frequency in Fig. 28 for comparison.

Fig. 29 shows the angular pulse distribution for a $100 \mathrm{GeV}$ shower (averaged over 50 showers) at $1 \mathrm{GHz}, 750 \mathrm{MHz}, 500 \mathrm{MHz}$ and $250 \mathrm{MHz}$ frequencies. The Gaussian half 


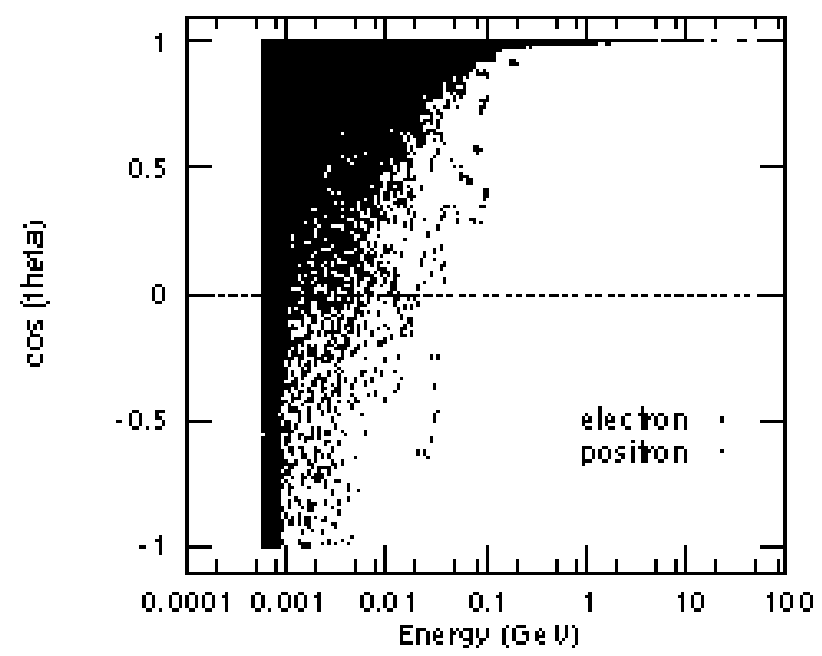

Figure 27: Plot of the cosine of the polar angle $(\theta)$ of each track-segment with respect to the shower-axis versus its total energy. Although high energy particles are very much aligned with the shower axis, there is an isotropic low energy component which moves with the shower. The isotropic component of the shower does not contribute to the Cherenkov pulse, which validates the removal of the particles when they fall below a certain low-energy threshold, say $0.611 \mathrm{MeV}$.

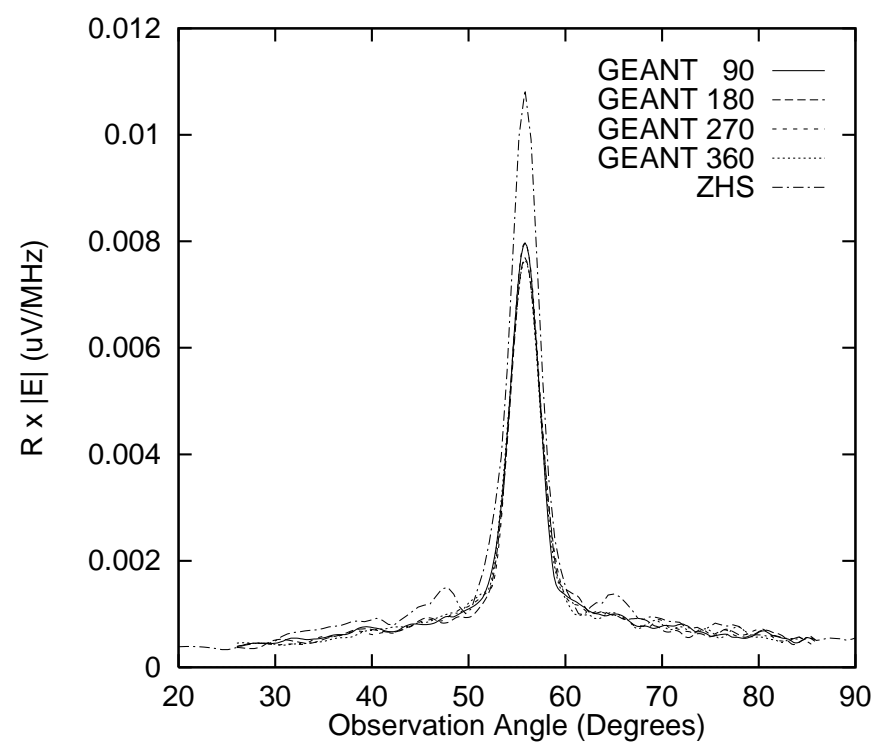

Figure 28: Angular pulse distribution of a $100 \mathrm{GeV}$ shower (averaged over 50 showers) with 0.611 $\mathrm{MeV}$ total energy threshold from GEANT. The pulse is calculated at $1 \mathrm{GHz}$ frequency and at 4 different azimuthal angles $(\phi)$. The Cherenkov peak at the observation angle $\theta=\theta_{c}$ shows very little dependence on $\phi$. The same pulse from the ZHS Monte Carlo is also plotted for comparison. 


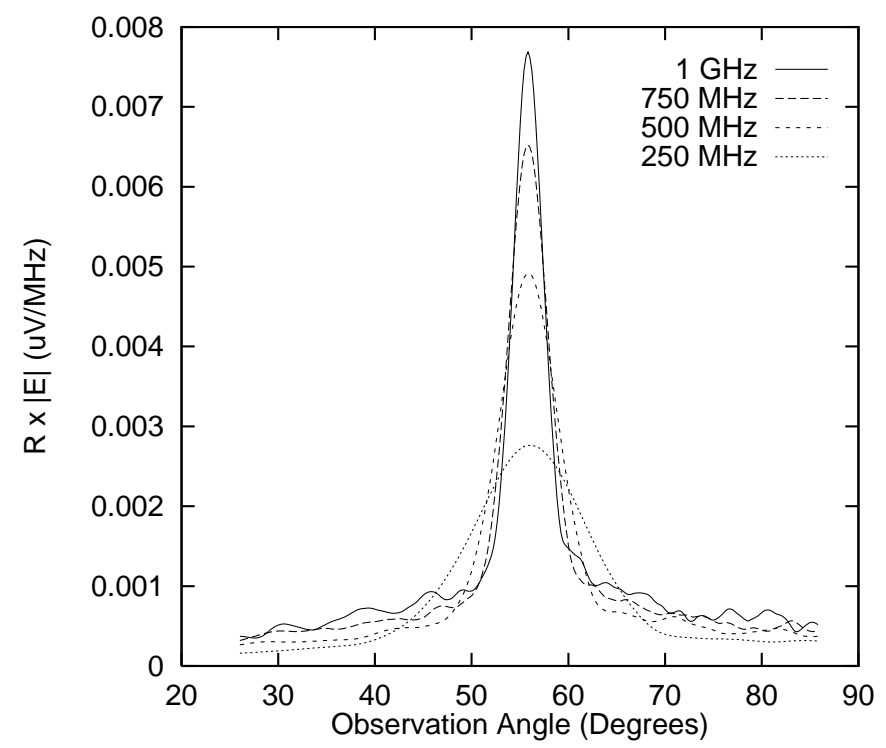

Figure 29: Angular pulse distribution of a $100 \mathrm{GeV}$ shower (averaged over 50 showers) with $0.611 \mathrm{MeV}$ total energy threshold from GEANT. The pulse is calculated at frequencies: $1 \mathrm{GHz}$, $750 \mathrm{MHz}, 500 \mathrm{MHz}$ and $250 \mathrm{MHz}$. The plot shows inverse scaling of Gaussian half width of the Cherenkov pulse with frequency.

width of the pulses are $2.0^{\circ}, 3.7^{\circ}$ and $7.3^{\circ}$ at $1 \mathrm{GHz}, 500 \mathrm{MHz}$ and $250 \mathrm{MHz}$ frequencies respectively. This corresponds to approximate inverse scaling of pulse width with frequency which is analogous to a single-slit diffraction pattern as pointed out in reference [9].

\section{Summary of Results and Conclusions}

We have analyzed $100 \mathrm{GeV}-1 \mathrm{TeV}$ electromagnetic showers and the radio frequency radiation they produce in great detail. These studies are a necessary ingredient for designing an experiment for radio detection of UHE cosmic ray induced showers in radio- transparent media. In particular, experiments to detect radio emission from showers induced by high energy cosmic ray neutrinos interacting in the surface of the moon 45] and in the South Polar ice-cap [46] are underway and have reported preliminary results. Coherent radio emission from electromagnetic showers has recently been demonstrated in the laboratory [12]. The technique is gaining recognition as a powerful tool for particle detection. The thorough dissection and understanding of all the intricacies of the showers and their relationships to the final radio pulse produced is our goal achieved in this paper.

Energy information for each stage of every track is readily available from the GEANT shower code. This information is essential for tracking the energy distribution in the shower and identifying the sources of radio emission. Among our results, is the direct determination of the radiation length in ice from an exponential fit to bremsstrahlung radiation energy loss as a function of depth in Sec. 3.1. When this information is combined with the direct extraction of the ionization loss, Sec. 3.3, we determined the critical energy 
from the data shown in Fig. 5 . The value obtained is nicely consistent with that found by using the Moliere radius extracted from the data for the radial energy flow, Sec. 3.2, in combination with the radiation length. The consistency between the critical energy values indicates that our application of the code and analysis of the data gives a correct physical picture of the interplay among the competing processes in the shower as it develops.

Because the coherent radio emission of interest depends upon the charge excess in the shower, we need the energy profile of the contributions to the charge excess. This again requires the GEANT track-by-track energy information, and we show the total charge imbalance broken down into energy ranges in Fig. 12. A large fraction, more than $50 \%$, of the imbalance comes from the energy range below $5 \mathrm{MeV}$. Though the track lengths are small, the large number of particles leads to a significant contribution to the total track length of the shower.

The total and projected track lengths for both the total and excess charge populations are determined and shown to be proportional to total shower energy in Sec. 3.5. The total track length is comfortably below a rough estimate of the upper bound for the total track length. Our detailed study of the longitudinal profiles in iron shows overall agreement but differences in detail among the GEANT, the EGS4 and the ZHS simulations in Fig. 8. The profiles in ice for $100 \mathrm{GeV}, 500 \mathrm{GeV}$ and $1 \mathrm{TeV}$ electron and photon induced showers in ice are well described by a modified Greisen parametrization with critical energy value extracted from the simulation data, as described above, and two fit parameters. The confidence levels of the fits are typically $80 \%-90 \%$, as summarized in Tables $1 \& 2$. The GEANT profiles in ice are qualitatively similar to those from the ZHS code, but lie typically $25 \%-35 \%$ lower. To determine whether small differences in cross section values used in the different simulations could account for this difference, we developed a 1-dimensional shower code with the full set of cross sections for the relevant processes. As we report in Sec. 3.7, the depth at maximum and the number of particles at maximum are rather insensitive to changes in the cross sections. We therefore believe that the differences in cross-sections are not the source of profile and total track length differences between our simulation and that of ZHS. Unfortunately, one important check that we have not been able to make is the GEANT vs. ZHS ionization energy loss $(\mathrm{d} E / \mathrm{d} x)$. The ZHS code does not admit a readout of energy loss by shower particles. Therefore, we were unable to make from ZHS code plots similar to Figs. 3 \& 1 we made with GEANT. If the ionization loss in ZHS code is much lower than in GEANT, it might account for the difference between showers produced by the two Monte Carlos.

We develop the framework to calculate the electric field in the $100 \mathrm{MHz}$ to multi $\mathrm{GHz}$ frequency range in Sec. 5. The track-by-track field calculation, applicable in the Fraunhoffer zone, and the calculation treating the shower as a continuous current density, applicable in the Fraunhoffer and and Fresnel zones, complement each other. We present both for this reason. The direct, numerical calculation of the total field from the vector sum of the fields from individual tracks allows a detailed study of the dependence on total track length. Moreover, we elucidate the effects of random direction changes due to collisions and the pattern of phase relationships among the contributions from all tracks. Complementing these intensive numerical calculations, we present analytic work that employs the form factor of the effective current density to calculate the field in both Fresnel 
and Fraunhoffer regimes. Given our realistic model for the current density, which we fit to the transverse charge distribution from the simulation, we show the remarkable result that the radiation from the shower is coherent over the whole shower at the Cherenkov angle in the Fraunhoffer limit.

Our study of the track-by-track phase coherence in Sec. 6 reveals that the phase associated with the initial time and position of a track (TP) and the phase associated with the "diffractive emission" (CP) from the track as a whole are uncorrelated. This result supports our model of the current, and consequently of the field, in Sec. 5. Figs. $17 \& 18$ show the strong phase peaking of the "diffractive" phase, called Cherenkov phase (CP) in the discussion, on and off the Cherenkov angle as defined by the shower axis. The phase associated with the initial coordinates of the track, called the translation phase (TP), is coherent at the Cherenkov angle but completely random away from that angle.

We noticed some time ago [47] that the frequency spectrum of the electric field at the Cherenkov angle, calculated directly from the track data and the Fraunhoffer zone formulas developed in Sec. 5., flattens out at frequencies above $2 \mathrm{GHz}$, as shown in Fig. 24. It is clear from the figure that the ZHS simulation shows the same behavior but they did not examine the high frequency region further [9]. We employ the form factor, which correctly accounts for the transverse spread of the shower and its effect on the field to analyze the frequency spectrum. We evaluate its Fourier transform, $F(\omega)$, two independent ways and find agreement. We then show that the observed spectrum of the field is faithfully represented up to $5 \mathrm{GHz}$ for $100 \mathrm{GeV}$ showers and up to $15 \mathrm{GHz}$ for 500 $\mathrm{GeV}$ and $1 \mathrm{TeV}$ showers. Up to $15 \mathrm{GHz}$, we now have a clear picture of the behavior of the frequency spectrum, which continues to show coherent behavior.

The electric field in the Fraunhoffer zone as a function of the observation angle from the shower axis peaks at the Cherenkov angle. The width of the peak shrinks inversely with frequency and the height (field strength) rises linearly with shower energy. These features confirm the ZHS results and the off-Cherenkov angular dependence is also similar. The height of the peak at the Cherenkov angle for a given energy and frequency is larger in their case by about 25-35\%. This is perhaps not a surprising difference between two independent shower simulations and field calculations. We have performed an extensive set of tests and cross-checks to validate our results.

\subsection{Conclusions}

Our study quantifies coherent Cherenkov radiation from high energy showers and shows that coherence persists from $100 \mathrm{MHz}$ to tens of GHz. The existence of Coherent Cherenkov radiation goes back to Askaryan, and has been studied for decades, while the persistence of coherent emission at the Cherenkov angle at multi-GHz frequencies is new. The persistence of coherence is established by our new, highly detailed study of the actual shower currents and corresponding phase distributions in CP and TP introduced in Sec. 6. The recognition that the TP is coherent over the whole length of the shower at the Cherenkov angle is a new insight into the connection between shower particles and the fields they produce. We have analyzed the electromagnetic shower characteristics in great detail, including the energy distributions and energy losses, so that a complete set of shower 
parameters can be extracted from the simulation data to confirm and cross check the consistency of our picture. A grand summary of pertinent parameters and comparisons with the ZHS and other sources where available is presented in Table 5.

Table 5: Comparisons between $100 \mathrm{GeV}$ showers in ice from GEANT and from ZHS Monte Carlos

\begin{tabular}{lcc}
\hline \hline Parameter & GEANT & ZHS \\
\hline Total Energy Threshold $(\mathrm{MeV})$ & 0.611 & 0.611 \\
Total absolute track length (meter) & $399 \pm 5$ & 642 \\
Total projected $(e+p)$ track length (meter) & $374 \pm 4$ & 519 \\
Total projected $(e-p)$ track length (meter) & $70 \pm 8$ & 131 \\
Position of the shower max. (radiation length) & 6.5 & 7.0 \\
Number of particles $(e+p)$ at shower max. & $111 \pm 7$ & $155 \pm 10$ \\
Excess electrons $(e-p)$ at shower max. & $20 \pm 2$ & $37 \pm 3$ \\
Fractional charge excess at the shower max & $\sim 18 \%$ & $\sim 24 \%$ \\
Cherenkov peak at $1 \mathrm{GHz}$ (Volts/MHz) & $7.5 \times 10^{-9}$ & $1.0 \times 10^{-8}$ \\
\hline \hline
\end{tabular}

The simulation and field calculation developed are directly applicable to energies up to $1 \mathrm{TeV}$. Higher energy showers can be "bootstrapped" by evolving the shower particles into this regime with a corresponding multiplication in particle number and emitted field intensity. We intend to parametrize and extrapolate the data to the multi-PeV data needed for the UHE showers relevant to the RICE experiment. With the GEANT base, it will also be interesting to investigate similar shower and field calculation for the hadronic component of neutrino-induced showers.

\section{Acknowledgement}

Help and advice from Enrique Zas at various stages of this work were invaluable. Discussions and working sessions with Jaime Alvarez-Muniz were essential to our understanding of the workings of the ZHS code. We thank George Frichter and Florian Hardt for early diagnostic works using the ZHS code. Comments and questions from Frances Halzen on this topic over many years have been most useful. Thanks to Tim Bolton for helping with initial set up of the GEANT Monte Carlo code. This work is supported in part by the NSF, the DOE, the University of Kansas General Research Fund, the RESEARCH CORPORATION and the facilities of the Kansas Institute for Theoretical and Computational Science.

\section{References}

[1] G. A. Askar'yan. Sov. Phys., JETP14:441, 1962.

[2] M. A. Markov and I. M. Zheleznykh. Nucl. Inst. Meth., A248:242, 1986.

[3] A. L. Provorov and I. M. Zheleznykh. Astropart. Phys., 4:55, 1995. 
[4] J. P. Ralston and D. W. McKay, in Arkansas Gamma Ray and Neutrino Workshop: 1989, edited by G. B. Yodh, D. C. Wold, and W. R. Kropp, Nuc. Phys. B (Proc. Suppl.) 14A 356 (1990); reprinted in Proceedings of the Bartol Workshop on Cosmic Rays and Astrophysics at the South Pole (AIP, NY 1990) Proceedings \#198.

[5] S. W. Stecker and M. H. Solomon. Space Sci. Rev., 75:341, 1996.

[6] F. Halzen and E. Zas. astrophys. J., 488:669, 1997.

[7] J. Rachen, R. Protheroe, and K. Mannheim. astro-ph/99080301, 1999.

[8] G. Frichter, D. W. McKay, and J. P. Ralston. Phys. Rev., D53:1684, 1996.

[9] E. Zas, F. Halzen, and T. Stanev. Phys. Rev., D45:362, 1992.

[10] P. B. Price. Astropart. Phys., 5:43, 1996.

[11] RICE Collaboration. Proceedings of the ICRC 1997. astro-ph/9709223.

[12] D. Saltzberg et al. Phys. Rev. Lett., 86:2802, 2001.

[13] CERN Publication. GEANT-Detector Description and Simulation Tool, 1994.

[14] L. D. Landau and I. J. Pomeranchuk. Dokl. Akad. Nauk., 92:535, 1953.

[15] L. D. Landau and I. J. Pomeranchuk. Dokl. Akad. Nauk., 92:735, 1953.

[16] H. Messel and D. F. Crawford. Pergamon Press, Oxford, 1970.

[17] A. B. Migdal. Phys. Rev., 103:1811, 1956.

[18] A. Misaki. Phys. Rev., D40:3086, 1989.

[19] J. P. Ralston and D. W. McKay, in High Energy Gamma Ray Astronomy (Ann Arbor 1990), APS Conference Proceedings No. 220 (AIP, NY, 1991) edited by J. Matthews.

[20] J. Alvarez-Muñiz and E. Zas. Phys. Lett., B411:218, 1997.

[21] for air shower applications of radio-detection, see: H. R. Allan, Progress in Elementary Particles and Cosmic Ray Physics, North-Holland, 1971. J. L. Rosner and J. F. Wilkerson, hep-ex/9702008. J. L. Rosner, hep-ex/9508011. J. L. Rosner, in First International Workshop on Radio Detection of High Energy Particles, RADHEP 2000 (Los Angeles), AIP Conference Proceedings No. 579 (2001) edited by D. Saltzberg and P. Gorham.

[22] H. Bethe and W. Heitler. Proc. Roy. Soc., A146:83, 1934.

[23] H. Bethe. Z. Phys., 5:325, 1930.

[24] F. Bloch. Ann. Phys., 16:285, 1933. 
[25] B. Rossi. High Energy Particles. Prentice Hall, 1952.

[26] W. Heitler. The Quantum Theory of Radiation. Clarendon, 1954.

[27] J. F. Carlson and J. R. Oppenheimer. Phys. Rev., 51:220, 1937.

[28] L. D. Landau and G. Rumer. Proc. Roy. Soc., A166:213, 1938.

[29] T. K. Gaisser. Cosmic Rays and Particle Physics. Cambridge University Press, 1990.

[30] K. Greisen. Prog. Cosmic Ray Physics, 3:1, 1956.

[31] A. M. Hillas. J. Phys., G8:1461, 1982.

[32] E. Fenyves, S. Balog, N. Davis, D. Suson, and T. Stanev. Phys. Rev., D37:649, 1988.

[33] J. Nishimura. Handbuch der Physik, 1967.

[34] W. R. Nelson et al. Phys. Rev., 149:201, 1966.

[35] G. Bathow et al. Nucl. Phys., B20:592, 1970.

[36] O. I. Dovzhenko and A. A. Pomanskii. Sov. Phys. JETP, 18:187, 1964.

[37] Particle Data Group. The European Physical Journal, C15, 2000. The details of EGS4 code system can be found in the report by W.R. Nelson, H. Hirayama and D.W.O. Rogers, SLAC-265, 1985.

[38] G. Grindhammer et al., at SSC Workshop on Calorimetry for the Supercollider (Tuscaloosa, 1989). SLAC-PUB-5072.

[39] J. D. Jackson. Classical Electrodynamics. Wiley, 1975.

[40] Fracis E. Low. Classical Field Theory. John Wiley \& Sons, Inc., 1997.

[41] L. D. Landau and E. M. Lifshitz. The Classical Theory of Fields. Pergamon Press, 1971.

[42] Florian Hardt. Unpublished, 1998.

[43] R. V. Buniy and J. P. Ralston. astro-ph/0003408, 2000.

[44] B. Rossi and K. Greisen. Revs. Mod. Phys., 13:240, 1941.

[45] P. W. Gorham et. al., in First International Workshop on Radio Detection of High Energy Particles, RADHEP 2000 (Los Angeles), AIP Conference Proceedings No. 579 (2001) edited by D. Saltzberg and P. Gorham.

[46] D. Besson, in First International Workshop on Radio Detection of High Energy Particles, RADHEP 2000 (Los Angeles), AIP Conference Proceedings No. 579 (2001) edited by D. Saltzberg and P. Gorham. 
[47] S. Razzaque. Talk given at Phenomenology for the Nu Century, PHENO 2000 (Madison) and at First International Workshop on Radio Detection of High Energy Particles, RADHEP 2000 (Los Angeles). 